\title{
IL RESTAURO ARCHITETTONICO AL POLITECNICO DI MILANO: L'APPORTO DI LILIANA GRASSI
}

\author{
AMEDEO BELLINI (*)
}

SuNTO. - Liliana Grassi, che opera a partire dal cruciale momento della ricostruzione post-bellica, deve affrontare nel restauro architettonico il problema dell'uso del linguaggio del modernismo, le cui basi concettuali si fondano sull'antistoricismo, muovendo sia dalla accettazione del nuovo linguaggio, sia dal rifiuto di considerarlo una sorta di "ultimo stile" formale separandolo dai contenuti sociali ed esistenziali che ne erano fondamento. Liliana Grassi rimane all'interno della tradizione che lega il restauro alla lettura storica dell'architettura da cui deduce parametri selettivi, ma in una visione che supera la vecchia posizione positivista, sfugge alle strettoie definitorie di arte e "non arte" della cultura neoidealistica. Con grande sensibilità progettuale realizza restauri di altissima qualità culturale e formale, riesce a far dialogare l'antico, recuperato con grande perizia tecnica, con il nuovo, come avviene nell'antico Ospedale Maggiore di Milano.

$$
* * *
$$

ABSTRACT. - Liliana Grassi, who works since the crucial moment of the post-war reconstruction, in the architectural restoration has to tackle the problem of the use of the language of Modernism, whose conceptual foundations are based on anti-historical authenticity, moving both from the acceptance of the new language and from the refusal to consider it a sort of "ultimate formal style", separating it from the social and existential contents on which it was based. Liliana Grassi stays inside the tradition that links restoration to the historical interpretation of architecture from which she infers selective parameters, but in a view that goes beyond the old positivist stand: she shuns the tricky definitions of art and "not art" of the neo-idealistic culture. With great planning sensibility she carries out restorations of cultural and formal excellence, she succeeds in making the old, recuperated with high technical skill, discuss with the new, as it occurs in the old Ospedale Maggiore in Milan.

(*) Istituto Lombardo Accademia di Scienze e Lettere, Politecnico di Milano, Italia. E-mail: amedeobellini@alice.it 
Una premessa necessaria alle considerazione su quale sia stato il contributo di Liliana Grassi ${ }^{1}$ al rinnovamento della riflessione sul

1 Le pubblicazioni di Liliana Grassi si segnalano, più che per il numero, per la qualità e la densità, si ricordano qui quelle relative al restauro architettonico ed a problemi di metodo storiografico. più pertinenti a quanto si dirà in seguito: Ambrogio Annoni, in "Palladio", ottobre-dicembre 1954; La Ca' Granda: storia e restauro, Università degli studi, Milano, 1958; Camillo Boito, Il balcone, Milano 1959; Storia e cultura dei monumenti, Società Editrice Libraria, Milano 1960 (all'interno di questa raccolta di saggi si segnalano, per la pertinenza agli argomenti qui discussi: Sulla tradizione; Sulla architettura "spontanea"; Il restauro dei monumenti, teorie e problematiche); Momenti e problemi di storia del restauro, Tamburini, Milano 1961; L'Ospedale filateriano e la sua nuova vita dopo i restauri realizzati per la sistemazione dell'Università degli Studi di Milano: criteri e metodi, in "Il monumento per l'uomo. Atti del Secondo Congresso Internazionale di restauro, Venezia maggio 1964", Marsilio, Padova 1971; Passato e presente nella conservazione dei centri storici, in "Vita e pensiero", settembre-ottobre 1975; Ideologia e memoria storica. Aspetti del rapporto passato presente nella cultura artistica e nel restauro, in "La situazione dell'uomo contemporaneo. Atti del convegno dell'Istituto Superiore di cultura religiosa”, S.l., s.n. ma Varese 1977; Restauro, in "Enciclopedia Universale", Unedi, Milano 1980; Problemi metodologici in relazione alla teoria del restauro, in "Il restauro delle costruzioni in muratura Atti del $2^{\circ}$ convegno Assirco”, Edizioni K, Roma 1981. Su Liliana Grassi il volume a cura di Maria Antonietta Crippa ed Emanuela Sorbo, Liliana Grassi. Il restauro ed il recupero creativo della memoria, Bonsignori, Roma 2007, con una antologia di scritti, la bibliografia completa delle sue opere e quella, sintetica ma esaustiva, di scritti che la citano: fra questi si possono segnalare: Amedeo Bellini, Maria Antonietta Crippa, Roberto di Stefano, Ricordo di Liliana Grassi, In "Restauro", n. ${ }^{\circ}$ 81, 1985; Maria Antonietta Crippa (a cura di), Liliana Grassi architetto: il pensiero, i restauri, i progetti, Assicurazioni Generali, Milano 1986; Lionello Costanza Fattori, Anna Maria Finoli, Commemorazione del membro effettivo Liliana Grassi, Istituto Lombardo Accademia di Scienze e lettere, Milano, 1986; Amedeo Bellini, La cultura del restauro 1914-1963, in "Il Politecnico di Milano nella storia italiana 1914-1963, Laterza, Bari 1988; Carolina Di Biase, Il rapporto con le preesistenze: $i$ problemi di conservazione e restauro nei programmi didattici, in "Il Politecnico di Milano nella storia italiana 1914-1963, Laterza, Bari 1988; Maria Antonietta Crippa, Liliana Grassi e il restauro: il fondamento realista e la continuità della tradizione italiana del restauro, in "Arte Cristiana", n. ${ }^{\circ}$ 782, Milano 1987; Amedeo Bellini, Liliana Grassi: un ricordo dopo dieci anni, in "TeMA", n. ${ }^{\circ}$ 4, 1995; Lucio Franchini, L'Ospedale Maggiore di Milano dedicato all'Annunziata detto la Ca' Granda, in "Lucio Franchini (a cura di) "Ospedali lombardi del Quattrocento. Fondazione, trasformazioni, restauri, New Press, Como 1985; Stefano Della Torre, Liliana Grassi, in Giuseppe Fiengo, Luigi Guerriero (a cura di) "Monumenti ed ambienti. Protagonisti del restauro del dopoguerra", Arte tipografica editrice, Napoli 2004; Amedeo Bellini, Liliana Grassi e la Ca' Granda, in "Il cuore della Ca' Granda. Ricordi, scoperte e nuovi temi di storia e restauro nell'Ospedale Maggiore di Milano (a cura di Maraingela Carlessi, Paolo M. Galimberti, Alessandra Kluzer), Cheiron, quaderni di aggiornamento storiografico, anno XXX, n. ${ }^{\circ}$ 59, Roma 2013. 
restauro architettonico nel Politecnico di Milano è la comprensione delle condizioni in cui la disciplina ed il suo insegnamento si trovavano all'inizio del suo affacciarsi nel mondo universitario con la collaborazione al corso di Ambrogio Annoni, figura dominante in quegli anni nell'ambiente milanese, le cui tesi nel dibattito, sono già stata altrove complessivamente delineate: ${ }^{2}$ qui le richiameremo per quanto necessario a porre alcune premesse. La sua posizione è stata spesso considerata ai margini del dibattito nazionale, proprio per le ragioni che la rendono attuale, ovvero per la sua scarsa adesione ad assunti precostituiti, per il rifiuto di schemi di riferimento di natura storica troppo ristretti, per l'opposizione della suddivisione in categorie del restauro, in rapporto allo scopo prevalente dell'intervento. Questo aspetto è stato messo in luce molto rapidamente quando sono iniziate le riflessioni sul suo pensiero, anche perché si collegava immediatamente ad una sua proposizione che aveva assunto un carattere identificativo, quasi uno slogan: operare individuando regole ed obiettivi di volta in volta, "caso per caso"; meno analizzato, se non recentemente, il suo rapporto con il progetto di architettura, ma si tratta invece della parte del suo pensiero, e della sua azione, che più ha inciso sulla pratica professionale nell'ambiente milanese, con riflessi di rilievo anche in ambiti più vasti.

La storiografia del restauro, ripetendo gli schemi di quella dell'architettura, ha individuato una successione temporale dei modi con $\mathrm{i}$ quali la disciplina si è manifestata, ciascuno progressivo rispetto al precedente: dal restauro stilistico, che ha come obiettivo il ritorno alla integrità originaria del monumento a quello filologico, che accetta la diacronia ed il carattere del monumento come testimone di una pluralità di epoche rifiutando come falsificazione della storia aggiunte in stile, ricostruzioni di quanto perduto. Questa tesi è prevalente fino al momento in cui i gravi danni bellici della seconda guerra mondiale ne hanno prodotto la crisi, di fronte ad una drammatica scelta: rinunciare a quei principi o accettare la perdita di una parte molto notevole del patrimonio architettonico. In realtà le distinzioni non sono affatto così nette: quei modi di operare, più che di teorizzare, si sovrappongono $\mathrm{e}$ sono tuttora presenti; la lettura storiografica si basava, essa stessa, su di una interpretazione progressiva della storia che quindi veniva letta

2 Amedeo Bellini, Ambrogio Annoni: arte e scienza dell' architettura, in "Annali di Storia dell'Università", Cueb, Bologna 2008. 
come una serie di successivi superamenti delle posizioni precedenti; in ogni caso le due tesi che qui si sono indicate, hanno al loro interno molte manifestazioni diversificate.

Più in generale osserviamo che fra le molte motivazioni a base del restauro nella modernità la più importante è la volontà di restituire il monumento alla sua funzione di documento veritativo della storia, che è esattamente quanto costituisce l'originalità del XIX secolo rispetto a quanto avveniva in precedenza, quando esso era motivato da intenti archeologici, da interessi antiquari, da esigenze utilitarie. E' l'applicazione all'architettura di quel complesso lavoro di analisi critica dei documenti, di qualsiasi genere essi siano, per depurarli dalle interpolazioni, dai travisamenti, per comprenderne totalmente il significato quando lacunosi. Presso taluni restauratori prevalgono il desiderio di unità stilistica, che non è soltanto espressione di un purismo formale, ma la volontà di esaltare il carattere educativo che il monumento assume con l'immediata suggestione dei suoi valori formali, a prescindere dalla cultura dell'osservatore; non a caso a metà ottocento in Italia la discussione intorno al restauro si intreccia con quella della ricerca e dell'esaltazione delle fonti di storia patria. La proposizione che richiede la conservazione delle stratificazioni di rilevanza storica ed artistica, dovuta principalmente a Camillo Boito, posta talora in totale contrapposizione con l'intervento di completamento formale, può essere letta in senso letterale o, come molte volte si è fatto, intendendone i termini secondo il significato attuale; in realtà ciò che si definisce valore storico-artistico è quanto rappresenta le qualità migliori della propria epoca. Siamo quindi all'interno di una idea progressiva della storia, della possibilità di identificare ciò che permette di tramandare il senso dei tempi, il mutamento positivo; una posizione che si unisce a quella, prevalente fra i restauratori, che riconosce un rapporto diretto tra società, condizioni sociali ed economiche, produzione d'arte, che è quanto giustifica il disprezzo per ciò che proviene da società corrotte, o che testimonia aspetti eticamente condannabili di un'epoca. ${ }^{3}$

3 Si pensi, per esempio, al giudizio del tutto negativo che in quel momento storico si dà di tutta l'architettura, ma non solo, del sei e del settecento, soprattutto di quest'ultima. Non è certamente possibile concludere che gli storici dell'arte considerassero quel momento storico totalmente negativo, ma invece si pensa e si scrive che l'arte definita con i termini "barocco" e soprattutto "rococò" rappresentava le caratteristiche negative e dominanti del tempo: retorica vuota, eccesso di decorazione, frivolezza, assenza di razionalità, esaltazione del superfluo. 
L'analisi storica, il riconoscimento degli stili e della loro successione, l'indagine sull'edifico da restaurare, il trarre elementi per la conoscenza generale e per il giudizio sul caso specifico, sono quanto viene definito "metodo storico", caratteristica peculiare della cultura italiana. L'espressione rappresenta bene quanto viene affermato nei primi strumenti di tutela: la circolare di Giuseppe Fiorelli, 1882; il documento approvato dal congresso degli ingegneri ed architetti italiani del 1883; i programmi e le proposte di regolamento delle attività di tutela dei direttori degli Uffici regionali per la conservazione dei monumenti, 1892, che accolgono le istanze diffuse presso i restauratori colti: eliminare ciò che appare deturpante, ricostruire ciò che è storicamente accertato, valersi della documentazione il più possibile accurata, non escludere il criterio di analogia, cioè di copia da monumenti coevi e congeneri. Le posizioni boitiane, proposte nel 1883, che non differiscono nelle linee generali da quegli obbiettivi di fondo, erano tuttavia potenzialmente dirompenti: la prescrizione di rendere riconoscibile, nell'insieme e nei dettagli, le parti nuove, con la nettezza che esprimono le sue parole, significava eliminare la regola dell'unità formale, dare un evidenza al restauro nel tessuto architettonico, dare la preminenza agli aspetti storici e filologici a scapito delle valenze comunicative, riducendo quindi quegli effetti didattici che uno degli scopi fondamentali del restauro. Il monumento "palinsesto" non poteva cioè avere quel carattere di immediata presa sull'osservatore che ne faceva strumento di persuasione. Si è spesso sottolineata una presunta incoerenza di Boito che avrebbe consentito numerose spoliazioni di monumenti, non tanto direttamente ma come persona frequentemente consultata anche dagli organismi statali

4 Il testo ed un esame ragionato dei singoli documenti in M. Bencivenni, R. Dalla Negra, P. Grifoni, Monumenti e Istituzioni, parte seconda, Alinea, Firenze 1992, in particolare pp. 4-41, 71-101.

5 Le indicazioni del documento proposto da Camillo Boito ed approvato dal congresso degli Ingegneri ed architetti italiani del 1883 giungevano a chiedere la distinguibilità delle aggiunte ad un monumento a qualsiasi scala, dai volumi aggiunti per ragioni funzionali, ai piccoli completamenti di sagome, a prescindere dal fatto che $i$ manufatti fossero il risultato di una attività artistica o avessero carattere geometrico. Un esempio probante è quello dell'eventuale integrazione delle parti di un elemento di architettura classica, cioè di una parte a carattere puramente geometrico, senza incertezze dimensionali, che viene prescritto sia compiuta con forme semplificate, e quindi in totale discontinuità. Vedi Atti del quarto congresso degli Ingegneri ed architetti italiani radunato in Roma nel gennaio del 1883, Centenari, Roma 1883, pp. 118-125. 
cui era affidata la tutela perché non si è tenuto conto dei limiti da lui stesso posti alla regola. e cioè quelli della rilevanza storico-artistica, nel senso già indicato. E' invece il principio di riconoscibilità che è stato largamente disatteso, e spesso anche quello della ricostruzione su basi storicamente certe, in nome della compiutezza logica e formale del monumento, del principio che fosse necessario presentare "un discorso conchiuso", per il quale poteva essere utile e sufficiente la verisimiglianza, il completamento logico delle premesse poste dalla riconoscibilità dello stile, realtà logica leggibile dall'esperto nei suoi valori fondamentali anche se lacunosa, e quindi presentata all'apprezzamento universale. Qui si pone la posizione di Luca Beltrami, figura per molti versi esemplare, i cui modi possono essere rappresentativi dell'atteggiamento di molti suoi contemporanei; il suo restauro venne definito come "storico", ovvero come un rifiuto del filologismo, ma anche dell'intervento stilistico che non apparisse fondato da un'ampia ricerca sul passato che fornisse al restauratore linee guida precise, che sono tuttavia una sua scelta che individua, tra i molti fatti di cui l'architettura è testimonianza, quelli che è opportuno "rivendicare" nel presente. Boito aveva in alcuni scritti proposto una suddivisione dei monumenti in rapporto all'epoca ed alle condizioni di potenziale uso: quelli archeologici destinati alla semplice contemplazione, per i quali era legittimo soltanto il consolidamento statico; quelli medievali, per i quali era necessario tener conto dei caratteri pittoreschi; quelli moderni, più vicini ai nostri usi e quindi suscettibili anche di qualche modificazione. Beltrami taccia le distinzioni proposte dal suo vecchio maestro come frutto di un rigidità burocratica che vorrebbe incanalare in schemi la ricchezza della realtà, la diversificazione dei casi e soprattutto la complessità del monumento, che non è mai univocamente definibile, non si presenta mai come appartenente ad una sola delle categorie indicate; questo atteggiamento corrisponde anche a quello di rifiuto di rigidi confini disciplinari, per esempio nel rivendicare la necessità di competenze di natura architettonica per gli archeologi. ${ }^{6}$

Secondo Beltrami il progetto di restauro non può essere condizionato da una serie di norme precostituite perché esso si basa sui rapporti effettivi del monumento con la storia che esso ha vissuto: non si tratta

6 Si vedano gli scritti in difesa di Giacomo Boni, in particolare Categorie che non reggono, in "Il Marzocco", 12 maggio 1929. 
di una ricomposizione che si valga esclusivamente del principio di analogia tra edifici coevi e congeneri, che comunque è guida fondamentale dei rifacimenti, neppure si deve giungere al ristabilimento dell'unità formale, che anzi è indicata come uno dei peggiori errori del restauratore quando prevalga su altri obbiettivi generali, ma di farne un'organica dimostrazione della storia che l'edificio testimonia, che nell'edificio si è svolta. Un'organicità del divenire letto dallo storico restauratore, che selezione cosa si debba presentare al pubblico, colto ed incolto. Quanto a noi può apparire una sorta di invenzione, o addirittura un fantasioso progetto della storia, non è tale agli occhi di chi lo produce perché egli si fonda su di una concezione deterministica che gli dona la presunzione di poter raggiungere la verità. Il linguaggio che si utilizza è quello dello storicismo eclettico, lo stesso che presiede alla produzione del nuovo, che si fonda sull'idea della continuità della storia, sulla tesi, espressa da Pietro Selvatico, che afferma la possibilità di utilizzare forme analoghe a quelle del passato in analogia di circostanze storiche. Il modo di operare di Luca Beltrami è quello prevalente nel mondo professionale, con variabile qualità, il punto di riferimento teorico è invece Camillo Boito.

Il primo dopoguerra è caratterizzato dalla presentazione aggiornata delle tesi filologiche da parte di Gustavo Giovannoni, che di Camillo Boito si proclama erede: ne riconferma i principi, li aggiorna, li approfondisce, li attenua in certe parti, li rende più rigidi in altre. Ma la pratica, come più volte si è constatato, non muta di molto nella sostanza: il completamento stilistico è frequente: ammesso nella teoria come eccezione, è consuetudine nella pratica. Lo stesso Giovannoni giunge a scrivere che se è vero che il "monumento" morto ammette il solo restauro di consolidamento senza integrazioni, non manca di sottolineare che tuttavia i monumenti morti talora possono tornare a vivere, con un esempio, quello dell'Abbazia di San Galgano, che darà luogo, al contrario, al più colto intervento di consolidamento archeologico di un rudere nella storia del restauro in Italia, opera di Gino Chierici.

L'immediato precedente a Liliana Grassi, il docente presso il quale ella si è inizialmente formata, Ambrogio Annoni, è personaggio che unisce una notevole semplicità caratteriale e di posizioni ad una quasi inestricabile complessità di implicazioni. Egli è allievo di Boito e di Moretti, ha collaborazioni con Beltrami che ricorda sempre con accenti di grande stima; non c'è dubbio che si debba considerare erede di quel metodo storico che non accetta rigide classificazioni, è costan- 
temente polemico verso quelle giovannoniane, ma è anche segnalato nella storiografia della disciplina come colui che, tra i primi, attua restauri in cui il rispetto per la stratificazione è dato caratterizzante. ${ }^{7}$

Il suo rifiuto delle suddivisioni categorizzanti, che, come ha osservato proprio Liliana Grassi, diviene un dato sistematico del suo pensiero sul restauro, assume le due posizioni contrapposte, filologismo e ricerca della compiutezza formale su basi storiche, come aspirazioni ugualmente legittime che si contemperano o prevalgono in rapporto ad una riconosciuta gerarchia dei valori presenti o sopravvissuti nell'edificio. Una assenza di rigidità che ha il ha il suo punto di forza in rapporto con il progetto del nuovo. E' tipico del restauratore filologico tendere comunque ad una compiutezza architettonica e sono note le perplessità, talora, come accade in Gino Chierici, fino ad una vera e propria angoscia, nel constatare quante siano le incertezze storiografiche, le responsabilità nel rapporto tra storia che si scopre, restauro che pone le condizioni con le quali il monumento sarà percepito in futuro, ma il metodo filologico di per sé escluderebbe un intervento "creativo", libero.

Il restauratore anatomizza il monumento, ne mette in luce le vicende, in molti casi esibisce gli elementi che lo compongono, il palinsesto, completa quando necessario con un intervento riconoscibile ma strettamente subordinato al testo. Annoni progetta sul monumento con lo spirito di chi si pone contemporaneamente dal punto di vista dello storico e dell'architetto che "compone", che mira a valorizzare la preesistenza. Nella pratica egli in taluni casi interviene con supporti modernissimi a reggere elementi antichi conservati a rudere, come avviene a Pomposa con un opera che non verrà compresa e quindi demolita, oppure nella basilica di Galliano con il rifiuto della ricostruzione dell'abside destra della basilica, ma anche con gli interventi apparentemente meno incisivi, ma non meno importanti, nella chiesa di San Pietro in Gessate dove si rinuncia all'unità formale mantenendo un portale rococò o di Santa Maria la Bianca del Casoretto, dove elementi secon-

7 Su Ambrogio Annoni si vedano: Amedeo Bellini, Ambrogio Annoni: arte e scienza dell' architettura, in "Annali di Storia dell'Università", Cueb, Bologna 2008; inoltre, anche per la conoscenza dell'ambiente in cui opera: Carolina Di Biase, Il rapporto con le preesistenze: i problemi di restauro e conservazione nei programmi didattici" in "Il Politecnico di Milano nella storia italiana", quaderni della "Rivista milanese di economia, n. ${ }^{\circ}$ 17”, Cariplo, Milano 1988 i vol. II, pp. 691-712; Amedeo Bellini, La cultura del restauro, 1914-1963, ivi, pp. 663-690. 
dari, come le inferriate di alcune finestre, sono inseriti con un linguaggio moderno, ma in sottotono, laddove neppure il più rigido dei filologi avrebbe contestato un intervento più mimetico. Altrettanto coraggioso il completamento della decorazione del palazzetto "veneziano" di Ravenna, dove un affresco moderno, di Adolfo De Carolis, assume la funzione di ripristinare una fascia di colore, di restituire un rapporto perduto tra decorazione ed architettura, reinterpretandolo. Al contrario, in altri casi, egli opera in modo ricostruttivo, esattamente secondo i termini del vecchio restauro stilistico, e si possono citare non tanto la Bicocca degli Arcimboldi, opera giovanile e con una qualche tutela di Luca Beltrami, ma piuttosto la Villa Mirabello, entrambi edifici di Milano. L'architettura di Annoni è legata al tardo eclettismo ottocentesco, sotto forme di un classicismo moderato, lontano dal rigorismo trattatistico, ma è pur sempre frutto di un pensiero e di un sentimento che si uniscono ad una sua capacità di comprendere anche quanto è fuori dalle sue tendenze: le aperture teoriche al moderno sono frequenti, e fanno parte di una sua generale attitudine alla comprensione dei fenomeni che gli stanno attorno. Lidea di fondo che lo guida è dunque quella del monumento come struttura viva, che deve vivere, che fa parte di un progetto generale dell'architettura, della città. E ciò lo rende apprezzato dai giovani architetti, i suoi allievi, che crescono nel clima del modernismo, lontani da ogni riferimento storicista. Non è qui il luogo di annotare i loro fraintendimenti, primo fra tutti quello che porta all'affermazione che il metro selettivo verso l'antico debba essere la sensibilità dell'architetto moderno che individua quella parte del passato che conserva validità per l'oggi.

E' forse utile ricordare che il numero delle persone che si occupano sul piano teorico di restauro dei monumenti è molto ridotto, come lo sono i luoghi in cui si elaborano le tesi la cui discussione caratterizza il panorama dell'immediato dopoguerra. I grandi argomenti che segnano il momento, fra di loro connessi, sono: le modalità di ricostruzione dopo i danni di guerra, sia per quanto riguarda il singolo edificio sia in rapporto all'ambiente urbano; la compatibilità della nuova architettura con quella antica; un clima culturale che vede, nell'ambito storico e del restauro, un affacciarsi, per molti versi un prevalere, della cultura critica neoidealista, in veste crociana o più genericamente di attualismo. La questione è apparentemente meno rilevante delle precedenti, ma in realtà in grado di influenzare atteggiamenti ed orientamenti, con l'esito di segnalare con forza valori della testimonianza storica non riconducibili al funzionalismo 
razionalista, ma anche concausa di una totale incomprensione tra protagonisti della cultura storica e progettisti, che prolunga il distacco che si era verificato con la separazione del dibattito sul restauro da quello più generale della nuova architettura. Si perpetuava una separazione originata dal fatto che nel restauro architettonico era stata prevalente la figura di Gustavo Giovannoni, uno dei protagonisti del rifiuto del modernismo, del controllo di regime sulla cultura architettonica, legato ad una visione positivista della storiografia architettonica, al cui fianco era schierata la maggior parte delle figure eminenti della disciplina. ${ }^{8}$

Un solo scritto di Annoni è dedicato al tema della ricostruzione, con intenti pratici e del tutto lontano dai temi più dibattuti; un fatto che può essere sinteticamente indicate nel carattere del personaggio, alieno dalle teorizzazioni astratte, conformemente alla tradizione dell'ambiente milanese, di cui egli è epigono, quella dei Beltrami e dei Moretti, che non ha mai separato la riflessione sull'architettura dal suo esercizio pratico, dalle considerazione sulla progettualità, dalle circostanze in cui i casi si presentano alla professionalità dell'architetto. Un modo di pen-

8 Schematizzando: il mondo del restauro appare legato ad una concezione vecchia dell'architettura, vicina al passatismo, chiusa di fronte al modernismo, orientata esclusivamente alla salvaguardia statica dell'eredità del passato, a fronte della quale si rivendica liberà di azione per il moderno. D'altro canto il modernismo è spesso accusato di non avere in sé un messaggio profondo, in grado di soddisfare esigenze di qualsiasi natura che vadano oltre agli aspetti materiali della vita, talora di essere strumento funzionale ad un economicismo speculativo che annulla di fatto qualsiasi finalità di ordine sociale. Non mancano equivoci da una parte e dall'altra: atteggiamenti poco colti o del tutto incolti, propensioni verso un intervento che si ritiene rispettoso della storicità attraverso la ricostruzione, preconcetti. Molto legata alla precedente la discussione sulla compatibilità del moderno accanto all'antico, sviluppata presso il cultori del restauro o della critica, con posizioni teoriche e conclusioni pratiche molto discordanti, opposte sia fra sostenitori di una compatibilità del moderno con l'antico, sia fra coloro che la negavano. Non è qui il caso di ripercorrerne i termini ma va ricordato che si va dall'affermazione di una sostanziale diversità del moderno dall'antico perché questo sarebbe una rappresentazione di un idea del mondo, mentre il primo si fonderebbe su basi esistenziali aspirando quindi a realizzare il modo del nostro stesso essere nel mondo, da arte figurativa a fenomeno esistenziale, fina al categorico rifiuto di questa tesi che nega il carattere unitario della storia, con la conseguente affermazione che al suo interno non si possa verificare una discontinuità.

9 Ambrogio Annoni, I monumenti milanesi danneggiati dalla guerra, in "Atti del Collegio degli ingegneri di Milano”, anno LXXX, n. ${ }^{\circ}$ 5-6, maggio-giugno 1947, pp. 66-74. 
sare del tutto consonante con una concezione della storiografia d'architettura che privilegia l'analisi degli organismi, dei tipi edilizi, della progressiva acquisizione della capacità di risolvere problemi costruttivi sempre più complessi, del rapporto, nei casi migliori, con l'evoluzione degli assetti sociali, ma anche questo in Annoni senza una particolare consapevolezza ideologica, ma con una notevole sensibilità architettonica. Una posizione legata quindi alla cultura del positivismo, alla concezione dell'arte come valore eteronomo che tuttavia non arriva ad essere storia sociale dell'arte. L'ambiente nel quale Annoni opera, il Politecnico degli anni della sua formazione come docente, è quello in cui domina un professionismo di variabile qualità, più o meno aggiornata nell'uso delle tecniche moderne, con orientamenti per nulla uniformi nella produzione architettonica, in cui il passaggio da un atteggiamento eclettico, proprio per esempio di Gaetano Moretti di cui tuttavia si apprezzano aperture verso certi aspetti del moderno, al monumentalismo pseudo moderno, al modernismo delle generazioni più giovani, avviene senza traumi apparenti, in una dimensione che sembra trovare la sua unità nei legami professionali, nell'appartenenza allo stesso gruppo sociale, ma anche ad una generica adesione ad una visione politica più che moderata nettamente conservatrice, con poche eccezioni. Confinati in altri atenei coloro che erano stati vicini al modernismo ed alle sue battaglie sociali, con tutti i limiti propri di personalità in cui la dimensione creativa e poetica supera quasi sempre gli assunti più propriamente programmatici della modernità, il clima culturale in campo architettonico è piuttosto mediocre. La vita della facoltà di architettura vive in sostanza secondo gli schemi nati con la sua fondazione, lontani dalla modernità, con una interpretazione mediocre e spenta delle ragioni ideali che pur presiedevano a quel progetto. La posizione di Annoni è tra le più dignitose: probo studioso, appassionato e brillante insegnante, colto, ai margini delle posizioni di potere, appare anche, e lo dimostrano le corrispondenze con la direzione del Politecnico, per molti versi insicuro, bisognoso di approvazione, di protezione, consapevole di appartenere ad un settore ritenuto del tutto secondario. Egli, pur nella relativa originalità della sua posizione sul restauro, partecipa del ritardo complessivo dell'ambiente milanese che si misura soprattutto nel sostanziale passatismo che lo caratterizza; nonostante ciò, alcuni aspetti del suo pensiero avranno una certa influenza sui modernisti, per usare uno schema riduttivo ma in questo caso chiaro, che ne accolgono, strumentalizzandole, le posizioni sul rapporto tra nuovo ed antico. 
Liliana Grassi inizia la sua attività universitaria con Annoni, verso il quale avrà in seguito parole sincere di stima, espresse anche nei colloqui con i propri allievi nei momenti in cui aveva occasione di rievocare i giorni della sua formazione. Ella si pone quindi nella tradizione del restauro che si fonda sulla conoscenza della storia, sulla individuazione di valori, con un atteggiamento di fondo selettivo: la figura dello storico- restauratore che risale alla impostazione data da Camillo Boito agli studi di architettura, perpetuata in termini nuovi, ma sostanzialmente riduttivi nella teorizzazione di Giovannoni e nelle conseguenze che se ne trassero sulla organizzazione degli studi, rimane. Ma i tempi sono mutati: il progetto di architettura si è emancipato del tutto dalla tradizione dell'eclettismo, del passatismo, si pone nel campo della modernità, se non del modernismo, deve affrontare problemi, impliciti ed espliciti, di grande rilevanza. Chi nella tradizione storica si era formato non può pensare che l'adesione al nuovo linguaggio debba determinare il totale rifiuto dell'eredità del passato, ma neppure che essa possa essere limitata nella sua possibilità di sopravvivenza dalle tesi della Carta di Atene dell'urbanistica, di Le Corbusier, ${ }^{10}$ subordinata alle esigenze della modernità, tanto meno essere considerata soltanto quando sia testimonianza di "valori plastici", quindi essenzialmente formali anche quando espressivi di una funzione. Ancora meno appariva accettabile un puro funzionalismo, sia come strumento per produrre il nuovo, sia come atteggiamento per affrontare il tema del rapporto con il passato. Si è posto l'accento sul giudizio che appare all'interno di documenti della facoltà: e cioè essere la Grassi la persona che aveva portato la filosofia all'interno del restauro, ${ }^{11}$ osservazione che deve forse essere chiarita sotto molteplici punti di vista: essa ha significato soltanto all'interno della facoltà di architettura di Milano, ove si ignoravano (non dalla

10 La Carta di Atene, documento approvato nel Congresso Internazionale di Architettura Moderna (CIAM) di Atene, 1933, attribuito sostanzialmente a Le Corbusier, pubblicato a Parigi nel 1941, anonimo per ragioni politiche, ripubblicato come opera del maestro del modernismo nel 1957, in Italia nel 1965 nelle edizioni di Comunità, Milano.

11 L'annotazione appare in un mio scritto Liliana Grassi: un ricordo dopo dieci anni, in "TeMA", n. ${ }^{\circ} 4,1995$, pp. 50-51, e fa riferimento ad un documento inserito nel fascicolo personale della studiosa, non più rintracciato; la memoria suggerisce che si trattasse di una presentazione di Piero Portaluppi, preside della facoltà, ad un concorso per la libera docenza. 
Grassi e da pochi altri) le fondamentali riflessioni di Roberto Pane ed in genere tutto il dibattito culturale interno all'area delle discipline storiche, ma va sottolineato che era un apporto, ritenuto giustamente rilevante, di una docente giovanissima, in un ambiente in cui la teorizzazione sull'architettura era sostanzialmente assente ${ }^{12}$ In secondo luogo deve confrontarsi con la posizione di Annoni, rilevante per le aperture che l'affermazione del "caso per caso" offriva alle determinazioni individuali del progettista, per quanto essa sottraeva il restauro alle ipoteche del passatismo, ma legata ad una conoscenza storica in parte empirica, in parte debitrice di un positivismo arretrato che non offriva un solido terreno a cui ancorare una moderna concezione dell'architettura. L'ambiente milanese, soprattutto interno alla facoltà, era attardato su posizioni storiografiche prive di un grande profilo, anch'esse di stampo positivista, ma senza le connessioni con le questioni che stavano alla base di un idea dell'arte come prodotto di una realtà sociale, ferme ad analisi tipologiche ed evolutive dei caratteri stilistici o costruttivi dei monumenti, o a raffronti ancor più descrittivi, compresi equivoci di tipo naturalistico.

Se la modernità di Annoni si esprime come rifiuto del decorativismo Liliana Grassi è pienamente consapevole del distacco dalla tradizione rappresentato dai principi su cui si fondava l'architettura moderna, dei contenuti fondamentalmente antistoricisti della nuova posizione, che non è congruente con la sua formazione culturale, con la sua forma mentis, con le sue aspirazioni spirituali. Chi, come Cesare Brandi, dichiarava il moderno incompatibile con l'antico, perché esso non rappresentava una visone del mondo, una poetica, il sentimento con cui l'artista percepiva ciò che gli era esterno, la riduzione del "mondo" alla sua interiorità, ma piuttosto la volontà di essere strumento dell'organizzazione esistenziale del mondo, non poteva corrispondere ad un idea di ciò che rimaneva per lei un'arte, un atto consapevole che non poteva rinunciare a valori monitori, alla trasmissione di dati ben oltre alla pura contingenza. D'altronde ella è consapevole che la separazione dell'architettura dai suoi contenuti

12 Sul piano specifico della progettazione del nuovo, della riflessione sulla funzione dell'architetto nel suo contesto sociale, sul tema di una moderna considerazione della storia che conducesse ad un superamento degli schematismi del razionalismo senza perdere i legami con i dati esistenziali, è contemporanea la riflessione di Ernesto Nathan Rogers, che sarà fondamentale nel rinnovo degli studi di architettura a Milano. 
sociali, dagli obbiettivi esistenziali che del moderno costituivano un fondamento essenziale si sarebbero confinate le nuove tendenze, una componente essenziale della cultura contemporanea, nella casella di una ennesima scelta formale senza contenuti specifici, altro stile nella storia degli stili. L'esigenza di comprendere a fondo l'integrità del progettare è fondamentale; Liliana Grassi, che prima di tutto si ritiene architetto a pieno titolo, orienta i suoi studi verso l'esame di un vasto orizzonte di temi e di epoche, alcune delle quali eccentriche rispetto agli studi al centro dell'attenzione in quel momento. Un arco di riflessioni molto ampio, che non risponde a scelte personali, a preferenze di natura estetica o di semplice gusto, all'esame di periodo storici privilegiati, ed ha invece lo scopo di considerare come si sia storicamente manifestato il fenomeno architettura ${ }^{13}$, nella produzione monumentale, nelle case di comune abitazione, nel suo inserirsi nel paesaggio, nel modo con il quale ogni momento era stato considerata dalla cultura contemporanea alla sua produzione e dalla critica successiva, nei suoi nessi con altre manifestazione della molteplice attività dell'uomo. Una verifica dei dati, un ripercorrere la storia, in testi filologicamente ineccepibili ma orientati molto più alla ricerca del significato dei fatti che non al loro accertamento, un riesame che prescinde da premesse ideologiche aprioristiche.

Il problema del rapporto tra "antico e nuovo", che pervade il dibattito degli anni del dopo guerra, la vede sostanzialmente estranea alle posizioni contrapposte in campo; la incompatibilità del moderno nell'antico non può rappresentare una soluzione: la presenza di valori "storici" sul territorio è tanto diffusa da rendere ineludibile il fatto; non c'è bisogno quindi neppure di affermare la continuità della storia da un

13 Mi sia consentito un ricordo personale: poche lezioni sull'architettura barocca in una breve supplenza al corso di Caratteri stilistici e costruttivi dei monumenti nell'anno accademico 1962-1963 furono sufficienti a determinare una revisione del giudizio negativo su quell'arte, frutto dell'insegnamento liceale e non scalfito dai corsi di Storia dell'arte e storia e stili dell'architettura dei primi due anni di corso universitario, fino a farmi sentire l'esigenza di un soggiorno a Roma per una vera e propria "immersione" nell'architettura berniniana e borrominiana. Alcuni anni dopo, all'inizio della collaborazione didattica, la sorpresa, certamente da attribuire alla mia ingenuità, nell'apprendere che il barocco "non gli piaceva". L'apprezzamento culturale, le valutazioni critiche acute e che avevano colto momenti fondamentali dell'interiorità di quella forma espressiva, l'interesse per il fenomeno, la volontà di comprendere nel profondo erano del tutto separate dall'apprezzamento formale; un atteggiamento che non è privo di conseguenze sulla questione del restauro. 
prospettiva di storicismo assoluto di origine idealista. Il problema, e l'esempio vale per molti casi, gli appare aperto, da verificare di volta in volta, ma sempre drammatico. L'analisi storica fondata, ovvero l'esame dei termini complessi con i quali si pone ogni problema, non è mai puro accertamento dei fatti che non appare essere mai il fine ${ }^{14}$, ma è piuttosto il motivo per delineare, comprendere, incrementare di contenuti il compito di progettare il nuovo, in una continuità che non è meccanica deduzione dal passato né tantomeno affermazione di un ineluttabile procedere della storia. Senza considerare questo, ed i tempi in cui maturano gli avvenimenti salienti del dibattito, potrebbe apparire marginale la posizione di Liliana Grassi soprattutto a fronte del grande dibattito del periodo post-bellico. Il suo sforzo di chiarire i contenuti dell'architettura, i suoi caratteri essenziali, il porsi quindi su di un piano critico generale non subordinato alla operosità ma anche a questa finalizzato, possono essere invece utilmente posti a confronto con quanto la critica delle arti figurative immediatamente precedente alla sue prime elaborazioni aveva detto in tema di restauro dei monumenti. Se prescindiamo infatti dal dibattito in ambito architettonico, che non trova riconoscimenti presso la seriosa critica delle arti figurative attardata in una visione di superficie degli organismi edilizi, con poche eccezioni che si riassumono nelle figure di Giulio Carlo Argan, Cesare Brandi e soprattutto di Carlo Ludovico Ragghianti, assai poco è dato riscontrare. E' bene ricordare, per un utile confronto, la scarsa qualità e le numerose contraddizioni che si riscontro nel capitolo sul restauro del Mentore di Ettore Modigliani ${ }^{15}$ quando tratta di architettura, a confronto con l'alta qualità critica complessiva di quell'opera, oppure l'inaccettabile banalità, sotto ogni punto di vista, delle lezioni di restauro architettonico di Roberto Papini ${ }^{16}$, critico per altri versi di eccezionali qualità.

14 Stefano Della Torre, Liliana Grassi, in Giuseppe Fiengo, Luigi Guerriero, "Monumenti ed ambienti. Protagonisti del restauro del dopoguerra", Arte tipografica editrice, Napoli 2004, pp. 72-86.

15 Ettore Modigliani [Fernanda Wittegens], Mentore. Guida allo studio dell'arte italiana, Hoepli, Milano 1941. L'opera era uscita con la falsa paternità dell'allieva dello studioso, per ragioni politiche, da lui stesso ricordate nella prefazione della seconda edizione del 1946.

16 Roberto Papini, Lezioni sul restauro dei monumenti tenute nella facoltà di architettura [di Firenze] durante l'anno accademico 1945-1946, dispensa universitaria s.e., [Firenze 1946]. 
Liliana Grassi pubblica nel 1960 una raccolta di saggi ${ }^{17}$ che costituiscono la testimonianza dell'attività didattica presso le Cattedre di Caratteri stilistici e costruttivi dei monumenti e di Restauro dei monumenti, in funzione subordinata ${ }^{18}$. Un esame dei campi esplorati è utile per segnare le differenze rispetto alle precedenze di Annoni, ma anche alle contemporanee operazioni sistematiche di Carlo Perogalli ${ }^{19}$.

Il volume si apre una premessa che ne illustra il significato, i limiti, la destinazione a persone colte non necessariamente specialiste dell'architettura, ma in cui soprattutto si afferma che esso costituisce un esame aperto dei problemi presi in considerazione, con la costante esposizione delle posizioni contrastanti, che l'autrice dichiara essere presente sovente, ma che in realtà è pressoché sistematica; ciò non per eludere l'impegno di assumere una posizione ma per lasciare all'ideale interlocutore, il lettore, la facoltà di scegliere; ma l'autrice è anche avvertita, ed esplicitamente lo afferma, che ognuno degli argomenti trattati, con l'ampiezza delle implicazioni che essi comportano, richie-

17 Liliana Grassi, Storia e cultura dei monumenti, Società Editrice Libraria, Milano 1960.

18 Liliana Grassi, laureata nel 1947, venne nominata assistente volontario presso gli insegnamenti di Caratteri stilistici e costruttivi dei monumenti e Restauro dei monumenti, tenuti da Ambrogio Annoni; nel 1948 è nella terna di idonei al concorso di Assistente di ruolo con Mario Salvadè e Carlo Perogalli, che venne prescelto da Annoni; libera docente nel 1956 è assistente incaricato a Caratteri stilistici del Monumenti nel 1957, nel 1958 di ruolo; nel 1959 ebbe a Milano l'incarico di Disegno dal vero ed ottenne l'idoneità in un concorso a cattedra di Restauro dei Monumenti della facoltà di architettura di Firenze che chiamò Piero Sanpaolesi; incaricata di Restauro dei Monumenti dal 1960 risulta vincitrice con Paolo Portoghesi e Manfredo Tafuri nel concorso a Cattedra di Storia dell'Architettura bandito dalla facoltà di architettura di Milano, che dopo aver chiamato Portoghesi utilizza un posto resosi libero per il trasferimento del professor Leo Finzi (Scienza delle costruzioni) alla facoltà di ingegneria istituendo la cattedra di restauro dei monumenti sulla quale chiama Liliana Grassi.

19 Si segnalano gli scritti più pertinenti ai temi qui trattati, fra la vastissima produzione di Carlo Perogalli, Monumenti e metodi di valorizzazione, Tamburini, Milano, 1954; La progettazione del restauro monumentale, Tamburini, Milano 1955; su Carlo Perogalli: Amedeo Bellini, Presentazione [della ristampa di "Monumenti e metodi di valorizzazione"], Guerini, Milano 1991; Carlo Perogalli: la tradizione del restauro nel pensiero di un architetto moderno, in "La fabbrica, la critica, la storia. Scritti in onore di Carlo Perogalli", Guerini, Milano 1993; Perogalli, in "Monumenti e ambienti. Protagonisti del restauro del dopoguerra", a cura di Giuseppe Fiengo e Luigi Guerriero, Napoli 2004, pp. 119-143. 
derebbe conoscenze specialistiche ed approfondite per una infinità di settori. Si manifestano così alcune caratteristiche che rimarranno costanti: la tendenza a verificare e comprendere tutto l'orizzonte delle posizioni possibili, la consapevolezza del carattere limitato e settoriale di ogni ricerca che affronti temi complessi, l'attitudine didattica di chi vuole offrire strumenti di conoscenza piuttosto che tesi predeterminate ${ }^{20}$ e di questo sono testimonianza anche le schede bibliografiche, utilissime ancora oggi.

I temi affrontati sono molti, e sarebbe impossibile illustrarli compiutamente ma possono essere ricordati mettendo i luce gli elementi che contribuiscono a definire il rapporto con quello centrale della sua attività didattica e professionale, il restauro architettonico.

Il primo è una esplorazione bibliografica relativa ad alcuni temi della critica architettonica contemporanea, principalmente il rapporto con la tradizione, tra dati formali ed estetici e funzioni sociali dell'architettura, vale dire alcuni dei nodi del dibattito postbellico sul moderno; in essi già si supera quella contrapposizione manichea che la storiografia aveva presentato tra modernismo e passatismo come contrapposizione tra formalismo e funzionalismo, tra socialità del moderno e carattere reazionario del suo rifiuto, in termini squisitamente sociali e politici, talora con accenti moralistici, che, se trovava parziali ragioni d'essere nella situazione italiana, non era certamente prospettiva storiograficamente priva di pecche ${ }^{21}$. Al tema della tradizione è dedicato il secondo saggio, di grande intensità critica, in cui, dopo un excursus storico, infine si esamina il rapporto tra modernità e storia che necessariamente si deve istituire muovendo da una riflessione sulle condizioni dell'attualità. Una prima osservazione è che se altri aveva affermato l'impossibilità di sostenere la discontinuità del moderno rispetto all'antico affermando l'inevitabile continuità della storia, con un fondamento nello

20 Non è insignificante ricordare che limiti e funzione degli studi contenuti nel volume sono esposti in maniera tutt'altro che discorsiva, ma piuttosto sistematica a sottolinearne il carattere metodologico.

21 Gli equivoci fondamentali sono dati dall'intreccio tra giudizi di natura formali e censure di ordine morale, ed è strano che esso appaia anche in testi ispirati al neoidealismo crociano, ove l'inevitabile esame analitico dei contenuti si traduce in valutazione negativa sul piano della qualità architettonica che è positivamente giudicata quando esprime la poetica del modernismo in particolare in Bruno Zevi, Storia dell'architettura moderna, Einaudi, Torino 1950, rivista nel 1955. ${ }^{3}$ 
storicismo assoluto nel quale non poteva trovare posto una presunta frattura tra l'essere rappresentazione di un sentimento del mondo ed il volere essere la determinazione esistenziale del nostro modo di essere del mondo, risolvendosi la cesura nel valore d'arte che sublima le componenti pratiche nella sintesi tra forma e contenuto, nella Grassi la questione è posta nella sussistenza di un inevitabile rapporto umano, nel presente ma inevitabilmente nel passato che determina i modi dell'esperienza, nell'esigenza esistenziale di evitare l'alienazione da ciò che ci circonda, nella necessità di ritrovare legami anche con una tradizione popolare che è rifiuto della "tirannia di sistemi maggiori"22 con significativi riferimenti alla musica popolare ${ }^{23}$, al folclore, alle tradizioni ed alle manifestazioni che legano il ricordo, il mito, la coscienza del passato, all'attualità. Importanti, in ambito architettonico, i cenni a Boito ${ }^{24}$, alla sua ricerca del linguaggio architettonico della modernità per l'Italia unita, al legame che nel progenitore dell'impostazione filologica del restauro si istituiva tra ricerca formale, e non solo architettonica, con la cultura sociale, con una adesione alle tesi più vive al suo tempo. Liliana Grassi avverte che una strada che prescindesse dal rapporto con il contesto storico e sociale avrebbe significato ricondurre il moderno al formalismo; affermata la necessità di un rapporto con la tradizione, la necessità di chiudere un solco spesso artificioso, teorizzato e non prati-

22 Liliana Grassi, Storia e cultura dei monumenti, Società Editrice Libraria, Milano 1960, p. 34. Una posizione che si manifesta compiutamente nell'opera Province del barocco e del rococò. Proposta di un lessico biobibliografico di architetti in Lombardia, Ceschina, Milano 1966, con evidente riferimento ed altrettanto evidente contrapposizione alla pubblicazione del volume di André Chastel, I centri del Rinascimento, Rizzoli, Milano 1965, con una infedele ma significativa alterazione del titolo originale Renaissance mèridionale, Gallimard, Paris, 1965 (alla redazione del volume di Liliana Grassi ha dato un contributo rilevante Gianni Mezzanotte).

23 I riferimenti escono dalle esemplificazioni architettoniche per allargarsi, per esempio, al folklore, alla musica, citato Bela Bartok.

24 Molto significativa la pubblicazione dedicata a Boito, visto nell'ottica di un precorrimento del moderno, ovviamente più sul piano del metodo che non dell'espressione architettonica, testimonianza di un momento specifico della cultura milanese del momento, soprattutto nella ricerca di una continuità tra moderno e tradizione, anche per il superamento delle rigidità del funzionalismo, con interessanti connessioni con la figura di Ernesto Nathan Rogers; condivisibili su questo piano le parole di Stefano Della Torre, Liliana Grassi, in Giuseppe Fiengo, Luigi Guerriero (a cura di) "Monumenti ed ambienti. Protagonisti del restauro del dopoguerra", Arte tipografica editrice, Napoli 2004, p. 72. 
cato, per esempio nei numerosi casi in cui l'architetto moderno tradendo le fondamentali premesse esistenzialiste e fenomenologiche del modernismo esprime l'intenzione di "creare" opere d'arte, termine a cui nessuno rinuncia, soprattutto progettando ambienti museali che quasi sempre rispondono a concetti e premesse di natura idealista.

Appartiene quindi ad un percorso coerente il saggio dedicato all'architettura "spontanea", titolo che viene messo immediatamente in discussione in una analisi che tende al chiarimento dei termini, dei significati attribuiti alle parole con le quali variamente si è definita l'architettura priva di connotazioni monumentali, potremmo meglio dire in cui sono assenti evidenti volontà rappresentative; l'analisi mentre mostra l'insufficienza di ogni tentativo di definizione, esplora la ricchezza del fenomeno, mette in luce taluni aspetti dell'architettura antica, medievale soprattutto, nel loro rapporto con l'attualità. Altrettanto significativo il saggio sulla tipologia che rifugge da qualsiasi organizzazione cronologico- evolutiva della storia dell'architettura, sia in senso tecnicista sia come esito di uno storicismo che presupponga l'inevitabilità del progresso: i caratteri stilistici e costruttivi dei monumenti quindi non sono in alcun modo esaminati come il mutamento del lessico architettonico, delle forme storiche, dell'organizzazione distributiva o della complessità tecnico costruttiva di tipo positivista, punti di vista ancora robustamente presenti nell'ambiente milanese, con varia qualità. Si tratta piuttosto dell'analisi degli organismi in rapporto ad una complessità di temi in cui si privilegia l'esame dello svolgimento della vita, del soddisfacimento dei bisogni elementari dalla risposta ad una regola religiosa, all'organizzazione domestica all'ideale spirituale che diviene matrice degli spazi. Temi che sono i punti fondamentali dell'esplorazione dei due capi successivi: gli edifici di culto, materializzazione delle aspirazioni spirituali, della proiezione verso la trascendenza; gli edifici dell'abitazione, con un nuovo confronto tra dati materiali ed espressione formale, la ricerca delle basi culturali e delle propensioni poetiche dei protagonisti dell'architettura a fronte della risposta reale ai bisogni della persona; un'indagine che trova il suo coronamento nello studio dedicato alla tecnica, cruciale per il modernismo, dove ancora una volta sono presenti sottili valutazioni sul rapporto tra mezzi e fini in architettura, espressi anche nell'esame delle finalità della decorazione; qui si rovescia l'ordinamento dei saggi precedenti e l'autrice muove dal moderno all'antico. L'argomento è cruciale sotto diversi punti di vista perché coinvolge il rifiuto da parte del modernismo della decorazione, in 
forma più o meno radicale, la ricerca della rappresentazione della funzione, dei valori formali ottenuti attraverso il disegno degli elementi costruttivi, il che riallaccia l'argomento a quello della tecnica.

Va notato che nel modernismo si verificano tendenze che vorrebbero l'architettura del tutto autosufficiente rispetto all'apporto delle arti figurative e quelle, all'opposto, che vorrebbero una integrazione delle arti che derivi comunque da una progettazione globale e totalizzante, prodotto dell'architetto o da una collaborazione fra artisti. Un atteggiamento che tende ad escludere il fruitore se non quando eventualmente sia partecipe del processo progettuale, fatto che può avere soltanto carattere eccezionale e contrasta con la destinazione di massa del prodotto edilizio, la più frequente. Soprattutto nella casa di abitazione, ma non solo, al di là di ogni studio di razionalizzazione, di valutazione di efficienza, dell'istituzione di parametri oggettivi di verifica dello sfruttamento dello spazio, il dato fondamentale è l'uso da parte di persone che pervengono nell'alloggio con un loro bagaglio di ricordi, di conoscenze, di costumi di vita del tutto legittimi, non omologabili alle utopie architettoniche, alle "poetiche" anche quando non ne abbiano trascurato le valenze, alle volontà figurative, nel peggiore dei casi alle ubbie dell'architetto. Sono anche un ricordo personale le molte opposizioni di Liliana Grassi a ogni concezione pervasiva del progettista, all'idea di una regolamentazione globalizzante del fenomeno edilizio, soprattutto negli aspetti più legati alla organizzazione personale della vita. Questi saggi pongono in luce in particolare la volontà, e la capacità, di esaminare i temi propri dell'architettura nella loro dimensione storica con lo scopo della comprensione del senso della sua presenza oggi. Le bibliografie ragionate che corredano le dissertazioni sono una compiuta ricognizione sullo stato delle conoscenze, non nella loro generalità, ma negli aspetti più attuali; esse ritrovano $i$ fili direttori che avevano condotto al dibattito in atto, alle scelte moderne dell'architettura, al di fuori da schemi di rigido storicismo o di un meccanicistico procedere per ragioni di cause ed effetti, con una capacità di sintesi critica rara, per chiarezza e ricchezza espositiva. Essi appiano talora privi di una presa di posizione e ciò dipende dal fatto che l'autrice ha chiara la vanità, l'irrilevanza etica di uno schieramento di parte, inevitabilmente ideologico, a confronto con la ricchezza dei casi, con la necessità di assumere di volta in volta decisioni che si fondino su di un giudizio critico circostanziato; la capacità di esaminare le articolazione della storicità dell'architettura in tutta la loro ampiezza non elimina però l'affermata necessità di un atteggiamento selettivo. 
Il volume sull'architettura barocca "minore" è in questo senso molto rappresentativo ${ }^{25}$ : esso offre un panorama articolato dell'attività architettonica del XVII e XVIII secolo, tendenzialmente esaustivo, grazie ad una indagine molto estesa delle fonti, ponendo in luce non soltanto le architetture eccezionali, che sono riconosciute come tali, ma anche quelle che connotano borghi e paesi, la cui "spiegazione", il cui valore storico, non risiede soltanto nelle qualità formali, ma anche nel fatto di essere parte di un ambiente, di una realtà sociale, testimonianza di capacità diffuse di fare con arte, come valore rappresentativo di una identità. Quindi non i grandi circoli intellettuali protagonisti del Rinascimento, tuttavia più volte da lei indagati, non lo svolgimento delle teorie e la loro applicazione, ma la diffusione delle capacità costruttive e del gusto, il rapporto tra le grandi invenzioni della ricerca spaziale e le loro declinazioni diffuse.

I limiti dell'indagine critica di Liliana Grassi sono in parte quelli della cultura milanese e quindi dell'ambiente in cui si era formata, e che tuttavia ella supera largamente. Alle spalle, e parliamo di storiografia e critica architettonica di cui sono protagonisti architetti, si registra una tradizione legata al positivismo, talora con gli equivoci di un determinismo naturalistico e puramente descrittivo, talora più felice nell'apprezzamento dell'architettura nelle qualità distributive, tecniche e costruttive, come organismo razionale, ma assai meno come testimonianza di un vivere civile, nelle sue più disparate e complesse dimensioni. Si era perduta, con poche eccezioni, la migliore qualità della storiografia del positivismo ottocentesco: quella che si riallacciava alla storia sociale dell'architettura. Ciò che Liliana Grassi supera sono i limiti di prospettiva culturale, in estensione e qualità, e con essi lo schematismo nel definire il rapporto tra storia ed intervento nel restauro: il suo tentativo è quello di addentrarsi nello studio del significato profondo dell'architettura, del suo senso come manifestazione complessiva della cultura, sorretta in questo da una vasta e approfondita conoscenza della letteratura, da una attitudine ala riflessione filosofica, da una rara capacità di cogliere l'essenziale delle questioni. Ma soprattutto Liliana Grassi deve affrontare un problema che non si poneva ad Annoni ed ai suoi contemporanei, e cioè quello di un linguaggio moderno dell'architettura, in un clima culturale non semplice. In que-

25 Liliana Grassi, Province del barocco e del rococò. Proposta di un lessico biobibliografico di architetti in Lombardia, Ceschina, Milano 1966. 
gli anni il rifiuto dell'eclettismo, così come posto dalla storiografia del contemporaneo, così come sentito dagli architetti, aveva un significato politico, che si presentava, potremmo dire, come una particolare caratterizzazione di un problema etico; fare architettura "moderna" significava anche lottare per la democrazia, per la soddisfazione dei bisogni sociali, per una programmazione economica di cui l'urbanistica si sentiva come parte determinante, per una eliminazione dei fenomeni di diseguaglianza che le difficoltà postbelliche avevano accentuato; non è qui il caso di ricordare contrapposizioni, arretratezze culturali delle parti contrapposte, strumentalizzazioni in buona e mala fede, proprie della situazione politica italiana, soprattutto quando si discuteva di prospettive di rinnovamento istituzionale, tutti temi trattati in altre e più competenti sedi; limitiamoci a ricordare che in campo architettonico si sono fronteggiati il più totale rifiuto per una pratica urbanistica che non fosse separata da criteri più generali di controllo del territorio, da una edificazione di fatto programmata da interessi speculativi, da un totale disconoscimento dei problemi sociali e culturali connessi all'architettura il che ha avuto anche l'effetto di far apparire degne di credito le più strane proposte, le più improbabili posizioni, che avevano come unico fondamento aspirazioni sentimentaloidi confuse, ora con l'espressione di una poetica ora spacciate per avveniristiche proposte economico-sociali. Una situazione che non ha mancato di riflettersi nella questione del restauro e della tutela, assimilate, quando positivamente prese in considerazione, ad un ambito proprio della cultura storica, un limite inevitabile al progetto, ma per lo più valutate come permanenza di quella opposizione al modernismo, eticamente condannabile, oggettivamente corresponsabile della prevalenza dell'azione speculativa. Le ben note vicende del tentativo fallito di Roberto Pane e di Giuseppe Samonà di avvicinare, o soltanto di far discutere, due mondi contrapposti, sono un documento esaustivo.

Liliana Grassi conosce bene il tema, oggetto anche di studi e riflessioni approfondite che escono dalla polemica per soffermarsi invece sul significato del razionalismo, nell'attualità, nelle sue manifestazioni in storiche in Lombardia come luogo di sperimentazione della ricerca nazionale, nell'architettura altamente formalizzata ed in quella rurale, anche nel periodo tra le due guerre. ${ }^{26} \mathrm{E}$ ' da questo sostrato che deri-

26 Si vedano, per esempio: Liliana Grassi, Razionalismo settecentesco e architettura neoclassica milanese, in "Aspetti, problemi, realizzazioni di Milano: raccolta di 
va l'affermazione della necessità etica per l'architetto di operare con le forme della modernità: al di là delle contingenze politiche l'architettura è espressione del proprio tempo, ne concretizza le istanze, utilizza i mezzi tecnici del suo momento. ${ }^{27} \mathrm{Ma}$ Liliana Grassi è anche alla ricerca di una espressone che non sia disgiunta dalla storia ed esprime quindi un'aspirazione che si registra anche presso chi, di altra generazione, cercava un accordo tra moderno ed antico provenendo da matrici passatiste. Scrive infatti la studiosa milanese che il porre mano ad un monumento delle rilevanza dell'antico Ospedale Maggiore implicava "in tutta la sua acutezza, il problema critico, vasto ed attualissimo, del restauro architettonico, della responsabilità verso la tradizione e della conseguente esigenza di un maggiore approfondimento del linguaggio figurativo moderno perché divenga sempre più comprensivo di quei valori culturali nei quali le forme nuove devono inserirsi. ${ }^{\prime 28}$ Si rileva qui, in un testo che si riferisce ad un intervento di restauro, quanto nella sostanza appare, direttamente o indirettamente, in molti altri scritti di Liliana Grassi, che potremmo definire di critica della storiografia, di riflessione sui temi che avevano pervaso il dibattito interpretativo di alcuni fondamentali momenti della storia dell'architettura. Non è possibile ripercorrere quei precedenti, complessi e ricchi di spunti precorritori, ma può servire alla nostra analisi il collegamento che viene istituito dalla studiosa con i momenti storici in cui è espressa un'esigenza di verità nel linguaggio dell'arte; adesione quindi al modernismo, nelle sue tensioni verso la sincerità strutturale, nella nettezza di definizione degli

scritti in onore di Cesare Chiodi”, Giuffrè, Milano 1957, pp. 289-305; Razionalismo architettonico dal Lodoli a G. Pagano, Bignami, Milano 1966. Quest'ultimo volume è costituito da due capitoli che esaminano la cultura del razionalismo architettonico settecentesco, il primo, mentre il secondo si sofferma su taluni aspetti del dibattito contemporaneo; la redazione si era valsa di molto materiale fornito da chi scrive queste note e da uno scambio di idee che rappresentò la prima esperienza di ricerca, ma anche una sorta di esame di ammissione nell'ambiente universitario.

27 Si veda: Liliana Grassi, Aspetti nuovi dell'antico Ospedale Maggiore sistemato ad uso dell'Università di Milano, in "Arte Lombarda", Alfieri, Milano 1955, pp. 136145, in particolare p. 137.

28 Ivi, idem. Analogamente Roberto Pane, dopo avere rifiutato l'idea di una discontinuità storica che impedisse l'accostamento del moderno all'antico, era tuttavia ostile ad meccanico inserimento del nuovo, per le sue caratteristiche puramente mercantili esigendo in linea teorica che fra i contenuti, in senso crociano, dell'opera che l'artista doveva sublimare nella sintesi artistica, fosse compreso il tema del rapporto con l'antico. 
spazi, nell'adesione alle funzioni ed alla loro espressione, che tuttavia non gli appare sufficiente a soddisfare quanto l'uomo richiede all'architettura, rimanendo quindi ben lontana dal funzionalismo.

E' infine opportuno considerare il tema del restauro dei monumenti, anch'esso all'interno del volume che abbiamo finora esaminato: vi si ripercorre una storia della disciplina in forme sostanzialmente simili a quelle consolidate, ma sono da notare alcune affermazioni sul rapporto tra restauri stilistici inventivi e quelli fondati su di un metodo storico di indagine che si concludono con l'esposizione sintetica e chiara delle tesi neoidealiste, che vennero tardivamente applicate al restauro architettonico nel secondo dopoguerra (come alla critica architettonica, ma non in forma esclusiva) quasi che gli architetti (dando ragione implicitamente a certe concezione del progredire storico) dovessero recuperare il tempo perduto ripercorrendo un sentiero già tracciato e consolidato verso la modernità senza poter prescindere da una fase, appunto quella neoidealista. La conclusione è piuttosto cauta: non sembra utile abbandonare totalmente norme e tendenze delle carte, come linee di orientamento. Mentre si accoglie l'idea che nuovo ed antico debbano restare unità distinte, autonome nella concezione formale, nulla si dice a proposito della tesi che il risarcimento del danno ed il completamento di elementi di ricomposizione dell'antico debbano superare il criterio della evidenza che non induca a fraintendimenti, e neppure si commenta il fatto che l'opera del restauratore di debba porre l'obbiettivo di creare un'opera d'arte nuova che comprenda in sé l'antica che fosse eventualmente pervenuta frammentaria. Ma ciò che pone la Grassi in posizione autonoma rispetto alla tesi idealista, le cui teorie fanno riferimento esclusivo all'opera d'arte, riconoscendo per ciò che non raggiunge quella qualità la correttezza della posizione filologica, è la conclusione in cui si tratta del passaggio dal monumento all'ambiente storico, un concetto che non è in alcun modo riconducibile al concetto idealista di opera d'arte. I riferimenti bibliografici e critici, l'analisi delle discussioni urbanistiche si svolgono in termini che non possono ridursi al filologismo né ad un idealismo astratto dalla realtà vitale. La conclusione è affidata a parole di Roberto Pane che esprimevano speranza per uno sviluppo armonico della nuova città accanto a quella antica, con un cenno al tentativo, fallito, di coinvolgere il modo dei progettisti in un dibattito che ponesse il tema dell'esistenza di un valore autonomo dell'antico, anche negli aspetti non monumentali, non suscettibile di strumentalizzazioni, economiche o ideologiche. 
Come è già stato osservato ${ }^{29}$ Liliana Grassi realizza nell'attività professionale, limitata ma estremamente significativa, le proprie convinzioni, e si tenga ben presente il fatto che ella si riteneva in prima istanza una progettista, appartenente a quella tradizione del restauratore-storico tipico della cultura italiana, legittimamente, osserviamo, ma in forma più aggiornata, tesa ad una visione globale del fenomeno architettura, che si pone problemi più complessi di quanto non fosse avvenuto in passato. L'illustrazione del suo contributo si può quindi completare con qualche nota relativa al suo principale lavoro, che ebbe ad impegnarla dall'inizio della carriera: il restauro dell'antico Ospedale Maggiore di Milano, a cui ebbe a dedicare più di una rilevante pubblicazione.

Un possibile restauro stilistico viene dunque giudicato una "interpretazione arbitraria e non pertinente alla realtà vera dell'edificio originario", ${ }^{30}$ quello filologico, cui non attribuisce caratteri di scientificità, gli appare frutto di una visone erudita e lontana dal significato etico dell'architettura, dalla tensione attuale che è quella che ricerca l'avvaloramento (un termine centrale nel pensiero di Annoni), il consolidamento, il rifiuto della ricostruzione, ovvero della falsificazione. Senza ripetere i notissimi dettati pratici che ne derivano, è importante sottolineare un quesito che Liliana Grassi si pone. Ella osserva che istituire un rapporto tra verità e modo di conservare pone il problema del limite da trovare tra due esigenze diverse: quella del rispetto del dato storico e quello del significato complessivo dell'opera, della sua comprensibilità, della sua funzione. E qui va ricordato anche il senso che il restauro ha sempre avuto sul piano didattico, per l'insegnamento della storia, per l'apprezzamento diffuso dell'architettura, per il significato che gli edifici assumono nella percezione dei luoghi della vita, per la costituzione della loro identità. Ma in questo caso "qual è l'orizzonte della verità? ${ }^{31}$ Quale il senso dell' integrazione che può avere valore sul piano urbanistico? ? $^{32}$ Quindi, per Liliana Grassi, piuttosto che la falsificazione, esito di una

29 Stefano Della Torre, Liliana Grassi, in Giuseppe Fiengo, Luigi Guerriero (a cura di) "Monumenti ed ambienti. Protagonisti del restauro del dopoguerra", Arte tipografica editrice, Napoli 2004, pp. 72-86.

30 Liliana Grassi, Motivi per una storiografia dell'architettura, Edizioni Universitarie Bignami, Vimodrone, 1956, p. 86.

31 Ivi, p. 87.

32 Qui si ponga mente a quanto già considerato sul tema del rapporto tra antico e moderno nella sua generalità. 
concezione volgare dell'architettura, è il rapporto con il contesto che può realizzare la conciliazione tra conservazione, rifiuto della musealizzazione, adeguamento alle richieste vitali; trovare la consonanza tra apprezzamento dell'antico e pratica del moderno. La continuità nella storia è realizzata nel mutamento, nell'integrazione, nella ricerca della sintesi. E' facile ritrovare ancora una volta questi motivi nella sua storiografia: nell' esame dei temi della frattura o della continuità nei momenti di grande trasformazione, della variabilità di quanto di volta in volta è considerato nuovo, del carattere unilaterale, e quindi in sostanza traditore, di ogni "metodo" di indagine o di operatività, della necessità nella situazione attuale di superare i limiti del "funzionale". ${ }^{33}$ Moralizzare l'architettura (che non può essere confusa con la speculazione, con la cattiva edilizia la cui esistenza non scalfisce la qualità del moderno) identificandola con la verità costruttiva non è sufficiente. Questa visione della studiosa può contribuire a dare senso più compiuto ad una contrapposizione che riproduce nell'ambiente milanese la incomprensione che divideva restauratori da progettisti. Da un alto si rivendicava, ed il protagonista della discussione era Lodovico Barbiano di Belgiojoso, la capacità ed il diritto dell'architetto, dell'artista, di sceverare quanto dell'antico fosse degno di sopravvivere, perché tuttora valido; un riconoscimento che spettava soltanto alla sensibilità di chi produceva l'arte nelle sue forme moderne. Un attualismo, probabilmente inconsapevole, ma in linea con una concezione di fatto estetica dell'architettura, lontana dall'impostazione funzionalista che tuttavia si rivendicava. D'altro canto, e qui Liliana Grassi, la rivendicazione dello storico che come tale ha la capacità di ritrovare il filo della continuità nel mutamento e che non subordina la sopravvivenza al proprio sentire poetico ma al giudizio critico, inteso con l'ampiezza che abbiamo intravisto; si ricordi che Liliana Grassi rivendica agli architetti la storia dell'architettura, purché in possesso di un metodo rigoroso, della necessaria cultura.

E' infine da notare che la studiosa non si disperde nel dimostrare come il suo intervento corrisponda ai principi consolidati del restauro, come avviene presso la maggior parte dei protagonisti del dibattito seguito ai danni bellici, quando le più incredibili ricostruzioni sono

33 Ivi, p. 75: “ ... la reazione contro le colonne e gli archi non significa reazione contro il Partenone o il Colosseo, ma contro le imitazioni del Partenone e del Colosseo." 
state giustificate, per esempio, con l'anastilosi (della polvere ...) e non si può certo dire che non avesse approfondito le questioni teoriche della disciplina ${ }^{34}$. La guida dell'operosità è nella storia, in un approccio progettuale in cui il moderno si afferma a fronte di una consolidata visione del passato continuamente rivista con spirito critico, senza che al termine si possa o si voglia dare il senso dell'attualismo neoidealistico. Non è davvero un caso che le qualità di architetto di Liliana Grassi si siano esaltate nelle opere che si collocano a fianco dell'antico, che lo completano o lo arredano, negli interni come negli esterni, senza la minima confusione di linguaggio. Un esame rapido delle "spiegazioni" che l'architetto dà del proprio operare ${ }^{35}$, che significativamente sono più estese quando rivolte al nuovo, può ulteriormente precisare. Nell'edificio dell'antico Ospedale Maggiore di Milano, danneggiato gravemente a seguito degli eventi bellici, ma con variabile grado di distruzione, quella quasi totale delle parte meno antica, di cui si afferma peraltro la scarsa rilevanza storica ${ }^{36}$, consente una liberà compositiva che può dare luogo ad una razionale soluzione degli spazi della soluzione prescelta, che mantiene lo schema a crociera, dopo la verifica che non ci fossero pregiudizi funzionali ${ }^{37}$, per coerenza tipologica, per dare continuità alla struttura fondamentale dell'organismo secondo l'orditura filateriana; non "ossequio" ma esigenza di mantenimento di una logica interna ${ }^{38} . \mathrm{Si}$

34 Anche uno degli interventi più discutibili, la ricostruzione dell'Oratorio di San Carlino alle Rottole, abusivamente demolito, non fu mai giustificata se non come fatto di valore morale, come evento che imponendo ai responsabili della demolizione costi notevoli poteva avere efficacia dissuasiva per eventi del genere.

35 Fra i diversi scritti dedicati al restauro della "Cà Granda" si vedano in particolare: Liliana Grassi, Lo 'Spedale di poveri' del Filarete, Università degli studi di Milano, Milano 1972; Aspetti nuovi dell'antico Ospedale Maggiore sistemato ad uso dell'Università di Milano, in "Arte Lombarda", Alfieri, Milano 1955, pp. 136-145; L'ospedale filaretiano e la sua nuova vita dopo i restauri realizzati per la sistemazione dell'Università degli studi di Milano: criteri e metodi, in "Il monumento per l'uomo - Atti del II congresso internazionale del restauro, Venezia 25-31 maggio 1964", Marsilio, Venezia 1971, pp. 545-548.

36 Liliana Grassi, Aspetti nuovi dell'antico Ospedale Maggiore sistemato ad uso dell'Università di Milano, in “Arte Lombarda”, Alfieri, Milano 1955, p. 136.

37 Ivi, p. 137.

38 Anche per rispetto delle propensioni di Annoni, (vedi: L'ospedale filaretiano e la sua nuova vita dopo $i$ restauri realizzati per la sistemazione dell'Università degli studi di Milano: criteri e metodi, in "Il monumento per l'uomo - Atti del II congresso internazionale del restauro, Venezia 25-31 maggio 1964", Marsilio, Venezia 1971, p. 546, in linea con le indicazioni del Consiglio superiore di Belle Arti. 
giudica il pensiero dell'architetto toscano realizzato fondamentalmente nello svolgimento in pianta; la semplicità "la felice monotonia" degli alzati trova riscontro in quelli moderni, volutamente rispettosi di un limite espressivo che non conduca a prevaricazioni sulla preesistenza, di cui rispetta la gerarchia spaziale, assumendo totale libertà laddove questa non risulta condizionata; ciò vale per l'espressione formale degli alzati, per le scelte di connessione fra i piani che definiscono l'involucro, nella ricerca del rapporto tra le penetrazioni di luce e le pareti, tra rigidità delle delimitazioni perimetrali e la definizione degli spazi interni subordinata alle necessità di movimento di numerose persone. La soluzione che vede gli orizzontamenti appesi alla copertura, con la riduzione degli ingombri delle strutture verticali, assume il carattere di una totale coerenza tecnica, costruttiva e formale con l'idea di conservare la fronte neoclassica su via Festa del Perdono, mantenuta ed essa stessa appesa, per sopperire alla sua fragilità, per dare all'interno la massima libertà distributiva. Questo progettare che dall'antico muove, con comprensione e rispetto, individua anche i limiti della ricostruzione: un lavoro complesso di ricerca dei materiali, di ricomposizione, di interposizione del nuovo necessario alla continuità fisica, ora come replica, ora come elemento "neutro" di ricongiunzione tra lacerti superstiti, ma sempre con il corretto criterio della prevalenza del materiale antico, della "certezza" filologica, un'opera condotta con grande scrupolo fino ai minuti particolari.

Lo stesso atteggiamento di fondo presiede alla ricostruzione del cortile della ghiacciaia, di epoca sforzesca: due lati sono ricostruiti con il materiale sopravvissuto, uno contribuisce a ristabilire il quadrato perimetrale con una suggestiva presenza a rudere, l'altro, è architettura moderna di cui sarebbe facile riconoscere i riferimenti alla fase della cultura del razionalismo in atto in quegli anni, totalmente diversa dal contesto ma con alcuni rimandi a dati formali propri degli elementi strutturali della vecchia costruzione, come l'alternarsi dei pieni e dei vuoti, la posizione delle ricorrenze orizzontali, gli interassi. Queste costruzioni possono essere efficacemente confrontate con quelle dell'ala moderna per riscontrare il progressivo affrancamento di Liliana Grassi dai condizionamenti del gruppo di lavoro; il progetto ha perso qualsiasi legame con i modi della tradizione culturale a cui faceva riferimento Piero Portaluppi ed appare coerente con alcune sistemazione interne presenti nell'ospedale, con altri lavori. Non diverso l'ultimo grande segno, la realizzazione dell'aula delle lauree, un'architettura 
"contenuta" dall'involucro antico, distinta, una stratificazione autonoma, che fu oggetto di violente polemiche in gran parte separate da una legittima divergenza sulla destinazione funzionale o sulle caratteristiche formali per assumere il carattere di un calunnioso attacco personale anche su aspetti che poco avevano a che vedere con le questioni del restauro, avendo invece radice in contrapposizioni accademiche, strumentalizzate per ragioni che in genere si definiscono politiche, termine che si potrebbe accettare soltanto nel suo senso più basso ${ }^{39}$.

Considerare la posizione di Liliana Grassi nelle sue espressioni più recenti non è cosa semplice, per una serie di motivi fra di loro intrecciati, non tutti direttamente riferibili in senso stretto alla elaborazione di tesi e proposte sul piano scientifico. Se per ciascuno è vero che gli orientamenti teorici non possono essere del tutto scissi dalle vicende personali, spesso ignote o trascurate, quanto meno nella scelta degli argomenti di studio e che comunque essi possono non emergere negli scritti, questo è particolarmente vero nel caso di Liliana Grassi: la ricerca della scientificità, la nettezza dell'esposizione, il rigore logico non sembrano lasciare spazio ad elementi estranei. Tuttavia gli avvenimenti politici che avevano caratterizzato i lunghi anni della contestazione qualche influenza sembrano averla avuta, quanto meno nell'impedire un confronto più diretto con i protagonisti di un rinnovamento delle tesi sul restauro, così come si sono manifestati a partire dalla metà degli anni settanta, nel considerare, quasi a priori, inaccettabili quelle proposte che apparivano provenienti da una ideologia materialista, marxista, o comunque connesse ad un pensiero politico totalitario. Un atteggiamento simile a quello diffuso nella vita politica, che portava a respingere ogni tesi, anche se potenzial-

39 All'episodio fa riferimento anche Stefano Della Torre, Liliana Grassi, in Giuseppe Fiengo, Luigi Guerriero (a cura di) "Monumenti ed ambienti. Protagonisti del restauro del dopoguerra", Arte tipografica editrice, Napoli 2004, p. 86, e nota 18 , con preciso riferimento alla pubblicazione: una vicenda molto più complessa di quanto possa apparire a prima vista, densa di retroscena in cui si nascondono: risentimenti professionali e familiari; lotte accademiche ben chiare osservando i legami del firmatario dell'articolo e soprattutto ricordando che in realtà nasconde altra persona; gli interessi, personali e partitici, di un personaggio politico di chiara scarsa onestà intellettuale, in seguito scomparso dalla scena. Lo "scandalo" provocò un allarme nella sezione milanese di Italia Nostra, la cui direzione, decisa in un primo tempo a condurre una battaglia a mezzo stampa, venne orientata da chi scrive ad una sorta di inchiesta: un accertamento dei fatti (vi erano state anche accuse di sperpero di denaro pubblico) e di verifica del progetto che si concluse con un documento di plauso. 
mente positiva, quando apparisse in grado di avvantaggiare una parte politica, di accreditare una visione complessiva, quello che sarà poi definito, con grande fortuna mediatica, l'effetto "K".

Non sappiamo come si sarebbero sviluppate le opinioni di Liliana Grassi la cui vita si interruppe poco dopo l'affacciarsi di tesi che tendevano al superamento del concetto stesso di restauro per proporre una conservazione integrale del dato materiale, che si sono presentate con maggiore evidenza nei secondi anni '70. Possiamo tuttavia proporre alcune considerazione muovendo proprio dagli sviluppi, per linee essenziali del suo pensiero, in cui sono presenti alcune costanti, caratterizzato da aspirazioni talora difficilmente conciliabili. Un dato ineludibile è l'aspirazione verso i valori della permanenza, di ciò che supera il transeunte per radicarsi nella coscienza in una continuità tra passato e presente; anche nelle prime riflessioni volte all'architettura estranea alla storia delle espressioni formali colte, è evidente la ricerca di valori, anche quando sorti da dati d'uso ed espressione di cultura materiale (termine estraneo al suo lessico) che si possano definire come parte di ciò su cui si è fondata la civiltà fino all'oggi. Con questo la ricerca del bello, del senso del bello, il superamento delle tesi del restauro fondate sul filologismo per una impostazione più ampia del problema critico. $\mathrm{Ma}$, si badi bene, e questa affermazione si fonda su conoscenza personale e su molte discussioni, senza alcuna propensione, inizialmente, per il restauro cosiddetto "critico" e per le tesi neoidealiste sull'arte, verso cui chi scrive propendeva. In effetti non era possibile conciliare una tesi che confinava il "restauro" come opera creativa alla rara presenza delle opere d'arte e confinava nei limiti del filologismo gli oggetti "altri”, istituendo una distinzione ontologica che il concetto di "letteratura artistica" non poteva sanare. E' sufficiente ricordare le molte esplorazioni problematiche della Grassi sulle espressioni "popolari”, di architettura "spontanea", sui centri storici e la profonda unità metodologica con le indagini sui fenomeni elitari o, se si preferisce, di "grande storia", per escluder ipotesi idealiste di qualsiasi natura. Tutto ciò accanto a propensioni politiche orientate, senza legami partitici e tantomeno a gruppi di potere, verso un liberalismo aperto alle istanze sociali, una socialdemocrazia (due correnti di pensiero che nei fatti non vedeva in contrasto), in ogni caso atteggiamenti francamente laici, impregnati da un forte senso dei valori di etica individuale e collettiva, della necessità della presenza delle istituzioni. La ricerca continua del "vero" non è separata dalla coscienza dei limiti della ricerca storica come si ritrova 
nell'affermazione reiterata che non sono coglibili nella loro totalità le ragioni "corrispondente alla verità", quelle per le quali una serie di fatti sono relazionati secondo una struttura, e quindi l'acquisizione che anche le "verità" consolidate possono essere teoremi non verificabili, nel tempo come nello spazio. E' chiara nelle sue considerazioni l'insufficienza di ogni valutazione dell'architettura che si limiti ad aspetti temporali, la storicità, o spaziali, l'artisticità e quindi i limiti di un'istanza puramente storica o puramente estetica, una concezione che con il tempo si rafforzerà passando dalla constatazione di un limite all'affermazione dell'esigenza del contemporaneo soddisfacimento delle due istanze. Riconoscere un certo grado di non verificabilità della storia, il riconoscere risultati non persuasivi nelle varie tesi espresse nel tempo sul rapporto tra passato e nuovo, comporta da un lato l'impossibilità di arrivare ad una compiuta scientificità, dall'altro l'esigenza di limitare l'arbitrarietà delle scelte: una via gli appare il rifiuto dell'esaltazione della personalità singolare dell'architetto progettista e di cogliere invece il senso della storia, né fabula né "ancella della poesia", nei suoi limiti, che sono quelli delle possibilità di conoscenza. Agire dunque entro uno svolgimento metodologico coerente, ciò che ella definisce un principio etico che riconosceva presente in Annoni la cui lezione era così colta in un senso ben diverso da quello letto dai suoi coetanei progettisti ${ }^{40}$. La conseguenza propositiva di questa impostazione, in cui è implicito il carattere comunque rischioso dell'agire, si esplicita con l'affermazione che l'intervento di restauro partecipa di un senso dell'avventura intellettuale, aperta, che non tollera "pianificazione" ovvero tesi dogmatiche preconcette; un concetto che unito alla sempre presente attenzione ai valori vitali assume un sapore di esistenzialismo positivo in cui la compresenza dell'obbligo di scelta e di perdita delle possibilità non produce angoscia ed inerzia; ma è importante sottolineare che ancora una volta è forte la presenza di istanze etiche.

Tuttavia si afferma spesso la rilevanza di un ritorno al concetto di opera d'arte, della presenza di una teoria della storia; il restauro è rappresentato come storia delle scelte critiche nel considerare il rapporto tra

40 Su questo si veda Amedeo Bellini, Ambrogio Annoni: arte e scienza dell'architettura, in "Annali di Storia dell'Università", Cueb, Bologna 2008. E' da verificare un nesso con l'imperativo etico kantiano che di operare con il massimo della conoscenza e della coerenza possibili. 
architettura, città come testimonianza di complessi fenomeni culturali, assieme di valori più ampio di quello della singola manifestazione costruttiva. Nelle opere analitiche si esaminano il concetto di storia nel tempo con le sottintese concezioni ideologiche, il concetto di arte, in cui si dà rilievo alle connesse concezioni politiche, sociali, religiose che dichiara tutte legittime, tutte limitate; il desiderio di conservare e tramandare è visto come volontà di coesistenza, di comprensione del senso relativo delle cose sul piano della storia. L'architettura è sempre analizzata nella duplice polarità: valore formale ed espressione estetica, condizioni di vita, ovvero espressione di individualità o di valori collettivi, ma è evidente una costante tensione verso la ricerca di una ragione universale.

In un testo che è riconosciuto da più voci come il più rappresentativo delle acquisizioni di Liliana Grassi ${ }^{41}$, si afferma essere il restauro “... una complessa opera critica che, proprio in quanto tale, trae il suo dato fondante dalla concezione della cultura, cioè della storia, dell'arte ..., dell'architettura.". Riservandoci di discutere fra poco il termine "critico" e le sue declinazioni osserviamo che con questo Liliana Grassi accetta che il restauro si manifesti in modi variabili nel tempo, assumendo come dato la cultura del momento in cui si realizza e con ciò l'impossibilità di "racchiudere in una definizione risolutiva ed univoca il principio primo del restauro giacché esso postula una scelta sulla legittimità di conservare la materia dell'opera, in quanto veicolo della forma, cioè in quanto immagine, oppure in quanto documento". Con questo è chiaro che quanto segue è la scelta della studiosa, la sua riposta all'imperativo etico di operare con i migliori strumenti culturali di cui ciascuno è in possesso, nel caso speci-

41 Restauro, in "Enciclopedia Universale Unedi: dizionario enciclopedico", Unedi, Milano 1980. E' da notare una affermazione che ne costituisce l'incipit: vi si afferma che alcuni aspetti tecnici del restauro sono comuni "per analogia" a quelli di ogni opera, architettonica, scultorea, pittorica e si esemplifica: patine, velature, lacune, conservazione dei materiali, tecniche di scavo, rilievi; prosegue "Anche parte della problematica generale elaborata per la conservazione di opere architettoniche può estendersi al restauro di dipinti o sculture, data l'imprescindibile esigenza dell'unificazione dei metodi, emersa dall'attuale riflessione critica in una visione unitaria del concetto di tutela”. Qui si ritrova un rovesciamento delle affermazioni più diffuse che vedono caso mai il restauro architettonico debitore di metodologie provenienti da quello delle opere d'arte figurativa, un implicito riconoscimento di prassi che si sono affermate sotto la spinta di tesi più rigidamente conservative, ma anche un affermazione ambigua sull'unità di metodo nella tutela che non è di per sé unità di metodo nella scelta delle tecniche ma neppure nelle ragioni che giustificano il restauro e tanto meno i metodi di restauro. 
fico di notevolissima complessità e con assoluta onestà intellettuale, come dimostra la successiva esposizione in quel volume del succedersi delle tesi del restauro. L'orientamento di Liliana Grassi si muove sempre più accentuatamente verso una concezione spiritualista, di rifiuto di ogni posizione fondata sull'utilitarismo, nell'affermazione delle componenti qualitative del dato architettonico, nell'accettare un'impostazione con fondamenti metafisici, nella ricerca dell'idealità, con un percorso intellettuale che non possiamo esaminare in dettaglio con molti punti di contatto, pur nella sua autonomia, con il pensiero di Rosario Assunto nella concezione dell'arte, della città, della storicità della natura antropizzata. L'esigenza di un pensiero "forte" diviene fondamentale. Alle sue parole posiamo affidare la conclusione: «La soluzione del problema dipende da una concezione globale del mondo, nella quale è fondamentale l'atteggiarsi nel o di fronte al tempo». In sintesi la fondazione filosofica e le motivazioni del restauro debbono rispondere all'esigenza di un tempo ritrovato; l'esigenza cioè “di fondare il tempo 'finito', 'necessario' della vita giornaliera e della storia nella sua transitorietà su un tempo 'infinito', 'duraturo'" un tempo ritrovato dell'arte che, come fu detto, non è reviviscenza storicamente già realizzata, né ritorno di un gusto o di uno stile come nei secoli scorsi. «Nel tempo ritrovato "L'uomo libera la propria temporalità" dalle catene della successione e recupera insieme il passato e il presente "in una realtà che non è attuale (e perciò più reale dell'attualità), in una idealità che non è 'astratta' e, perciò, più veridica dell'astrazione intellettuale” (Rosario Assunto)».

Liliana Grassi afferma infine che, al di là di sterili "polemiche tra avanguardia ed arretratezza" la questione "richiede il raggiungimento di una chiara consapevolezza teorica", da lei certamente raggiunta nella volontà di fondare il restauro su valori di trascendenza: una manifestazione della ricerca dell'essere contrapposta all'idea che il divenire sia l'unica forma di essere; resta un aporia, ineliminabile nella ricerca di un pensiero "forte" che il trascendente si traduca in operatività il cui valore è inevitabilmente inevitabile transitorio ${ }^{42}$; rimane il dubbio della

42 Calandoci per un attimo nelle questioni pratiche (ma il restauro è una pratica) anche Liliana Grassi riconobbe che certe sue rimozioni non erano state opportune, vedi la testimonianza di Stefano Della Torre, Liliana Grassi, in Giuseppe Fiengo, Luigi Guerriero (a cura di) "Monumenti ed ambienti. Protagonisti del restauro del dopoguerra”, Arte tipografica editrice, Napoli 2004, p. 76, e nota 17. 
legittimità della applicazione di concetti di trascendenza nel campo dell'operatività, ma è chiaro che questo porterebbe la discussione intorno al possibile carattere relativo della verità, concetto che si può ritrovare in taluni scritti lontani ma che non appartiene alle sue conclusioni finali. Ciò che appare "certo" è che il punto di arrivo del pensiero di Liliana Grassi sul restauro è "probabilmente" il più alto di quanto riferibile alle tesi sul restauro, più consapevole di quanto non siano celebrate e grandi (nel senso di essere un tentativo omnicomprensivo) teorie.

Un modo di concepire il rapporto con il passato che è stato definito "recupero creativo della memoria storica" ${ }^{43}$, una definizione che coglie sinteticamente un dato fondamentale del suo pensiero e che rende giustizia anche alla coscienza che di sé aveva Liliana Grassi, cioè di essere architetto, progettista, artista.

Un commento è d'obbligo sull'uso del termine "critico": applicato al restauro esso indica originariamente una posizione di stretta osservanza attualista, di una forma di idealismo che non è quello di Liliana Grassi, e che tuttavia ha come fondamento quel recupero dell'integrità dell'immagine a cui ella stessa fa talora riferimento, ma i cui principi pratici, per esempio l'irriconoscibilità dell'integrazione, ha sempre fermamente respinto. In seguito il termine ha avuto un uso più generale: intervento che si basa su di una analisi qualitativa e selettiva, spesso in polemica con tesi più strettamente conservative che rifiutano, e qui sta l'antitesi, selezioni basate su parametri estetici e storiografici, considerate perciò acritiche, mentre esse ritengono semplicemente di avere spostato il momento selettivo sul piano dei valori etici, a prescindere da concezioni spiritualiste o materialiste ${ }^{44}$. Già si è espresso il rammarico

43 Titolo della pubblicazione dedicata a Liliana Grassi da Antonietta Crippa, 2007, vedi nota 2 .

44 Il rinnovamento prodotto dalle tesi conservative, con un incremento senza precedenti degli accertamenti analitici e della esplorazione delle tecniche di conservazione materiale ha condotto ad atteggiamenti pratici meno selettivi, con la coniazioni dell'ossimoro "conservazione critica", anche se l'ossimoro è figura retorica che affiancando due termini inconciliabili genera un sentimento inesprimibile sinteticamente (una buio accecante ..., cioè annichilente) qui nel nostro caso è difficile immaginare. In realtà una consolidata prassi "storiografica", la cui buona fede andrebbe verificata, tende ad ignorare la sussistenza di una tesi "conservativa" distinta da quella restaurativa e con propri fondamenti concettuali per ridurre la conservazione a mera tecnica, si veda, ad esempio, la voce "Conservazione" nell'aggiornamento della Enciclopedia Treccani, Roma 19, a firma di Giovanni Carbonara, Roma 2015. 
per il fatto che la prematura scomparsa di Liliana Grassi abbia impedito un confronto culturale seren ${ }^{45}$, che avrebbe avuto certamente interesse per i forti fondamenti etici del suo pensiero e del suo atteggiamento di vita, mentre talune circostanze legate alle vicende politiche della facoltà di architettura avevano fatto assimilare quelle tesi al momento contestativo, ad atteggiamenti anti istituzionali, ad un materialismo pratico, non senza qualche iniziale fondato motivo.

Senza entrare in particolari che sconfinerebbero inevitabilmente in prese di posizione personali è opportuno segnalare alcuni dati estremamente positivi del carattere di Liliana Grassi: la grande indipendenza di giudizio che l'ha condotta ad assumere le posizioni che riteneva corrette a prescindere dal tornaconto che ne sarebbe potuto derivare in termini di attività professionali e di potere accademico; il rifiuto di adattare la sua attività didattica alle richieste che apparivano dettate da contingenze politiche; un senso molto alto del valore e del significato delle istituzioni, a tutti i livelli, che l'hanno talora condotta a rifiutare posizioni politiche di per sé corrette quando si presentassero come finalizzate a scardinarle $\mathrm{e}^{46}$. Tutto ciò in un momento in cui l'ambiente culturale in cui si era formata e all'interno del quale aveva ottenuto i primi importanti riconoscimenti era contestato ${ }^{47}$. Una rottura traumatica quando la

45 Stefano della Torre. Liliana Grassi, in Giuseppe Fiengo, Luigi Guerriero (a cura di) "Monumenti ed ambienti. Protagonisti del restauro del dopoguerra", Arte tipografica editrice, Napoli 2004, p. 81.

46 Un esempio molto pertinente (e fonte di infinita discussioni con l'autore di queste note) l'atteggiamento in occasione del referendum sul divorzio in cui avrebbe voluto vedere vincente la tesi abrogazionista.

47 E' opportuno ricordare che le prime forme di contestazione, manifestate a Milano fin dal 1963 avevano per oggetto fondamentalmente questioni relative ai programmi di insegnamento, particolarmente nel settore della composizione architettonica e dell'urbanistica che sottintendevano una figura professionale come soggetto in grado di avere un ruolo attivo nella definizione del senso dell'architettura nella società e non come mero esecutore di atti tecnici finalizzati al servizio di una committenza privata i cui compiti si esaurivano fondamentalmente nelle scelte formali. Una conseguenza di questo orientamento era anche quello di compiere attività di "ricerca" che avesse un significato culturalmente significativo sul duplice fronte della formazione personale e del servizio sociale. L'elenco delle richieste formulate in quel momento farebbe sorridere lo studente di oggi, per esempio quella di avere informazioni sulla formazione dei piani regolatori al di là delle norme tecniche (larghezza delle strade e profilo della loro schiena d'asino, larghezza dei binari tramviari ed altezza dei fili...); la loro apparente ovvietà è la misura delle carenze della situazione della facoltà in quel momento. In quel 
protesta assunse forme radicali di valore globale che travalicavano le questioni proprie della facoltà di architettura che tuttavia rimaneva un crogiolo di ogni sperimentazione, di ogni proposta criticamente fondata o politicamente coraggiosa, oppure frutto dell'ultimo libro venuto di moda, dell'espressione velleitaria di ogni bizzarria personale, di innovative concezioni sull'architettura ed il suo insegnamento. Ma tutto ciò anche, palestra di chi mirava alla propria posizione di potere ponendosi comunque "a sinistra" di qualsiasi proposta, talora con molta rozzezza; di chi cavalcando con abilità ogni momento contestativo si augurava di divenire il padre nobile della futura imminente palingenesi, non senza smentire in privato ciò che andava compiendo in pubblico; di chi tentava di dirigere rimanendo nell'ombra e fungendo da garante verso gli ambienti politicamente radicali ma ben lontani dall'auspicare l'arrivo di quella "Cina" che appariva sempre più vicina. Non mancava neppure chi agiva più radicalmente di altri con la foga del profeta in perfetta buona fede, e quindi con l'inconsapevole foga devastatrice di chi si ritiene portatore di una verità, soprattutto quando espressa con criptici messaggi collocabili tra l'ermetismo poetico e l'espressione dei buoni sentimenti di chi ama l'umanità ma non sempre gli uomini. Un insieme di fenomeni non facilmente districabile che ha lasciato tracce durature positive nel riordinamento degli studi e nel costituire una facoltà che pur fra errori ed incertezze ha realizzato una consapevolezza del corpo docente e degli studenti prima sconosciuta ma anche dato luogo a pratiche dequalificanti solo tardivamente eliminate, si è mossa per lungo tempo su di un terreno di assoluta illegalità ${ }^{48}$, di sostanziali pratiche

frangente non tutti i docenti avevano subito contestazioni dirette e riferite ai contenuti del loro insegnamento, e fra essi ovviamente i pochissimi le cui opinioni apparivano consonanti al nuovo clima, Ernesto Nathan Rogers primo fra tutti, e Liliana Grassi, nonostante che apparisse estranea al nuovo clima ma per la qualità del suo insegnamento, non assimilabile al mediocre professionalismo che caratterizzava la facoltà. Ovviamente fin dalla sua origine la protesta aveva connotazioni politiche, non erano mancate tentativi di strumentalizzazione né personaggi, fra gli studenti, che vi aveva fondato percorsi di carriera accuratamente programmati, ma si era ben lontani dal senso di radicale e globale rifiuto che prevarrà in seguito.

48 Il punto più basso forse fu quello che vide la facoltà occupata, agibile soltanto per quei docenti che avessero firmato un documento politico, con la connivenza se non con l'esplicita approvazione della presidenza, cui seguì la denuncia morale (e forse anche alla magistratura) di coloro che si rifiutarono di aderire, e l'avrebbero fatto anche se lo scritto avesse riprodotto loro parole, tacciati di assenteismo. 
totalitarie, di predominio delle valutazioni ideologiche. In quelle circostanze, che assunsero la loro forma estrema soprattutto poco dopo la chiamata in cattedra di Liliana Grassi, dovuta all'appoggio del Politecnico nel suo complesso, ad un indubbio prestigio, alla sua capacità di muoversi nelle difficoltà. Ella si trovò ad essere il possibile ago della bilancia fra due fazioni contrapposte paritarie nel numero e nella pretesa di monopolizzare il potere, teorizzando che fosse giusta prassi quella che una fazione maggioritaria assumesse su di sé tutte le responsabilità, pronta a cederle se divenuta a sua volta minoranza, a prescindere dal trascurabile fatto che in gioco vi erano posti di ruolo, per definizione inamovibili. In ogni caso Liliana Grassi aveva di fronte posizioni politiche che non poteva condividere, di per sé ritenute inaccettabili a prescindere dalle strumentalizzazioni in atto. Sarebbe stato facile assumere una posizione strumentale, ma la scelta fu quella di una quasi solitaria battaglia contro l'illegalità, fra mille manifestazioni ostili pubbliche e poco sostegno privato, anche da parte di ambienti esterni all'ambito universitario. Le vicende della facoltà consigliarono un passaggio a quella di ingegneria: pochi anni dopo iniziava quel processo di revisione che acquisendo gli elementi positivi della lunga crisi si riconduceva ad una condizione di sostanziale normalità. Si pose anche il tema di un possibile ritorno di Liliana Grassi che sarebbe stata possibile soddisfacendo la sua richiesta: che gli venisse dato formale e pubblico riconoscimento della correttezza della sua posizione passata, una condizione "irricevibile" per evidenti motivi politici che non gli potevano sfuggire. Una richiesta che presupponeva il rifiuto: non sarebbe mai tornata di fronte alle persone da cui era stata osteggiata, il cui atteggiamento aveva percepito ostile anche più di quanto oggettivamente non lo fosse, non distinguendo tra fatti personali e circostanze che implicavano e rendevano inevitabile un duro contrasto, per ragioni ideologiche sostenute in buona fede, per ragioni che spingevano a "cavalcare" gli avvenimenti per dominarli, per ridimensionarne il senso, trarne vantaggio, per tutte quelle cose insieme.

Questa impossibilità di recuperare un rapporto in cui fosse implicito il riconoscimento di ragioni e torti, anche relativizzando gli avvenienti, richiama un aspetto molto difficile del carattere di Liliana Grassi: estremamente dura nel difendere i propri spazi, tesa ad un lavoro solitario in cui ogni collaborazione poteva essere soltanto subordinazione, diffidente quanto più non si potrebbe di fronte ad ogni azione che potesse apparire interferenza, poco propensa a mettere in 
discussione quanto aveva elaborato a seguito, è bene dirlo, di un processo interiore rigoroso ed insieme tormentato. A questo si aggiunga una difficoltà ad affrontare direttamente e sul piano personale l'interlocutore di cui aveva compreso la strumentalità delle posizioni, per una particolare forma di timidezza, di rispetto umano, ma anche in non infrequenti casi, l'assunzione di posizioni pretestuose e "fuori tema" per non affrontare una situazione scabrosa, senza particolare riguarda per chi potesse esserne danneggiato. Un limite, come tutti ne abbiamo, accentuato dalle difficoltà della situazione, senza dimenticare che se indubbiamente il clima della facoltà abbattuta dalla contestazione era quello di un gretto professionalismo (senza dare effettiva padronanza agli allievi degli strumenti necessari) nella "nuova" (accanto a molte acquisizioni che costituivano una riforma duratura, che istituivano prospettive feconde, soprattutto nel rapporto tra didattica e ricerca, nell'introduzione nell'insegnamento di aperture sul sociale inevitabilmente connesse con l'architettura) non mancavano posizione strumentali in cui il "non farsi scavalcare a sinistra" copriva posizioni di professionalismo non meno gretto ed incolto, a prescindere dalle capacità di "creare" forme. ${ }^{49}$

49 Possiamo esemplificare, in termini ovviamente riduttivi ma che sarebbe facile ricondurre a valutazioni più generali. Nella vecchia facoltà: bandito l'insegnamento dell'urbanistica se non come tecnica applicativa (larghezza delle strade, curvatura della sezione, dimensione dei binari...) fino al caso dell'espulsione fisica di un assistente che aveva osato parlare di piano regolatore ("in facoltà non si fa politica"). Nella nuova, un celebrato docente, in un seminario, direttamente interpellato dagli studenti sulla questione dei centri storici ebbe a dire, e non sono troppo sintetico nel riferire, che taluni sostenevano un atteggiamento conservativo, altri di rinnovamento; il suo atteggiamento personale venne riassunto nell'espressione "io ci do dentro". Difficile conciliare queste affermazioni recise di un "io" creativo che non trova limiti nel contesto storico con la problematicità tormentata di Liliana Grassi. 